\title{
Approximation Algorithms for Reconstructing the Duplication History of Tandem Repeats
}

\author{
Zhi-Zhong Chen* $\quad$ Lusheng Wang ${ }^{\dagger} \quad$ Zhanyong Wang ${ }^{\ddagger}$
}

\begin{abstract}
Tandem repeated regions are closely related to some genetic diseases in human beings. Once a region containing pseudo-periodic repeats is found, it is interesting to study the history of creating the repeats. It is important to reveal the relationship between repeats and genetic diseases. The duplication model has been proposed to describe the history [3, 9, 4]. We design a polynomial-time approximation scheme (PTAS) for the case where the size of the duplication block is 1. Our PTAS is faster than the previously best PTAS in [4]. For example, to achieve a ratio of 1.5 , our PTAS takes $O\left(n^{5}\right)$ time while the PTAS in [4] takes $O\left(n^{11}\right)$ time. We also design a ratio-6 polynomial-time approximation algorithm for the case where the size of each duplication block is at most $2^{1}$. This is the first polynomial-time approximation algorithm with a guaranteed ratio for this case.
\end{abstract}

\section{Introduction}

The genomes of many species are dominated by short segments repeated consecutively. It is estimated that over $10 \%$ of the human genome, the totality of human genetic information, consists of repeated segments. About 10-25\% of all known proteins have some form of repeated structures ranging from simple homopolymers to multiple duplications of entire globular domains. In some other species, repeated regions can even dominate the whole genome. For example, in the Kangaroo rat (Dipomys ordii) more than half of the genome consists of three patterns of repeated regions: AAG (2.4 billion repetitions), TTAGG (2.2 billion repetitions) and ACACAGCGGG (1.2 billion repetitions) [11]. Recent studies show that tandem repeats are closely related with human diseases, including neurodegenerative disorders such as fragile X syndrome, Huntington's disease and spinocerebellar ataxia, and some cancers $[2,8]$. These tandem repeats may occur in protein coding regions of genes or non-coding regions. Since the initial discovery of tandem repeats [12], many theories on the biological mechanisms that create and extend tandem repeats have been proposed, e.g., slipped-strand mis-paring, unequal sister-chromatid exchange and unequal genetic

\footnotetext{
${ }^{*}$ Department of Mathematical Sciences, Tokyo Denki University, Hatoyama, Saitama 350-0394, Japan. Email: chen@r.dendai.ac.jp. Part of work was done during a visit at City University of Hong Kong.

${ }^{\dagger}$ Supported by a grant from the Research Grants Council of the Hong Kong Special Administrative Region, China [Project No. CityU 1196/03E]. Department of Computer Science, City University of Hong Kong, Tat Chee Avenue, Kowloon, Hong Kong. Email: cswangl@cityu.edu.hk.

${ }^{\ddagger}$ Department of Computer Science, City University of Hong Kong, Tat Chee Avenue, Kowloon, Hong Kong. Email: zhyong@cs.cityu.edu.hk.

${ }^{1}$ In the conference version, we wrongly claimed a ratio-2 polynomial-time approximation algorithm.
} 
recombination during meiosis (see [1] for details.) The exact mechanisms responsible for tandem repeat expansion are still controversial. Thus, the study of repeated regions in biological segments has attracted lots of attentions $[1,4,5,6,7,9,13]$.

\subsection{The Duplication Model}

The model for the duplication history of tandem repeated segments was proposed by Fitch in 1977 [3] and re-proposed by Tang et al. [9] and Jaitly et al. [4]. The model captures both the evolutionary history and the observed order of segments on a chromosome. Let $S=s_{1} s_{2} \ldots s_{n}$ be an observed string consisting of $n$ segments of the same length $m$. Let $r_{i} r_{i+1} \ldots r_{i+k-1}$ be $k$ consecutive segments in an ancestor string of $S$ in the evolutionary history. A duplication event generates $2 k$ consecutive segments $l_{c}\left(r_{i}\right) l_{c}\left(r_{i+1}\right) \ldots l_{c}\left(r_{i+k-1}\right) r_{c}\left(r_{i}\right) r_{c}\left(r_{i+1}\right) \ldots r_{c}\left(r_{i+k-1}\right)$ by (approximately) copying the $k$ segments $r_{i} r_{i+1} \ldots r_{i+k-1}$ twice, where both $l_{c}\left(r_{i+j}\right)$ and $r_{c}\left(r_{i+j}\right)$ are approximate copies of $r_{i+j}$ (see Figure 1). Assume that the $n$ segments $s_{1}, s_{2}, \ldots s_{n}$ were formed from a locus by tandem duplications. Then, the locus had grown from a single copy through a series of duplications. A duplication replaces a stretch of DNA consisting of several segments with two (approximately) identical and adjacent copies of itself. If the stretch contains $k$ segments, the duplication is called a $k$-duplication.

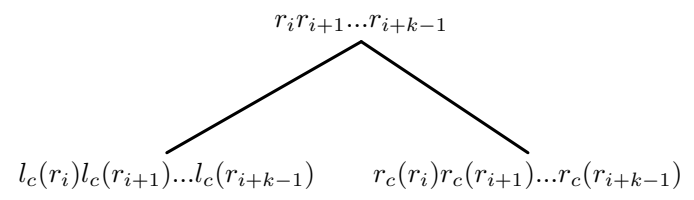

Figure 1: A $k$-duplication, where both $l_{c}\left(r_{i+j}\right)$ and $r_{c}\left(r_{i+j}\right)$ are approximate copies of $r_{i+j}$.

A duplication model $M$ for a string $S=s_{1} s_{2} \ldots s_{n}$ of $n$ tandem repeated segments is a directed graph that contains vertices, edges, and blocks as shown in Figure 2. Each vertex in $M$ represents a repeated segment (not necessarily in $S$ ), each directed edge $(u, v)$ indicates that vertex $v$ is a child of vertex $u$, and each block represents a duplication event. Certain edges in the model are allowed to cross each other; this is described using an edge-crossing rule (to be specified later).

Each vertex may have at most one parent and either zero or two children. There is only one vertex, called the root, that has no parent. The root represents the original copy at the locus. Those vertices that have two children are called the internal vertices, while the others are called the leaves. The two children of each internal vertex $v$ are distinguished as the left child and the right child of $v$, respectively. Each leaf is labeled with a segment $s_{i}(1 \leq i \leq n)$. Moreover, the left-to-right order of leaves in $M$ is identical to the order of the segments in $S$. If there is a directed path from a vertex $s$ to another vertex $t$, then $s$ is an ancestor of $t$ and $t$ is a descendant of $s$.

A block in $M$ represents a duplication event and hence consists of one or more internal vertices. Each internal vertex appears in a unique block; no vertex in a block is an ancestor (or descendant) of another vertex in the same block. The following rule applies to the children of vertices in each block $B$ : If the left-to-right order of the vertices in $B$ is $v_{1}, v_{2}, \ldots, v_{k}$, then their children are placed (under them) from left to right in the model in the order $l_{c}\left(v_{1}\right), l_{c}\left(v_{2}\right), \ldots, l_{c}\left(v_{k}\right), r_{c}\left(v_{1}\right)$, $r_{c}\left(v_{2}\right), \ldots, r_{c}\left(v_{k}\right)$, where $l_{c}\left(v_{i}\right)$ (respectively, $r_{c}\left(v_{i}\right)$ ) is the left (respectively, right) child of $v_{i}$ for each $1 \leq i \leq k$. Hence, for every two integers $i$ and $j$ with $1 \leq i<j \leq k$, the edges $\left(v_{i}, r_{c}\left(v_{i}\right)\right)$ 
and $\left(v_{j}, l_{c}\left(v_{j}\right)\right)$ cross each other in the model. However, no other edges cross in the model. For simplicity, we will draw a box to depict a block in $M$ only when the block represents a $k$-duplication event for some $k \geq 2$. We also refer to $k$ as the size of the block.

Each edge in $M$ carries a cost which is simply the hamming distance between the two segments associated with the two endpoints of the edge. The cost of $M$, denoted by $c(M)$, is the total cost of edges in $M$. We remark that all our results apply to other distance measures satisfying the triangle inequality.

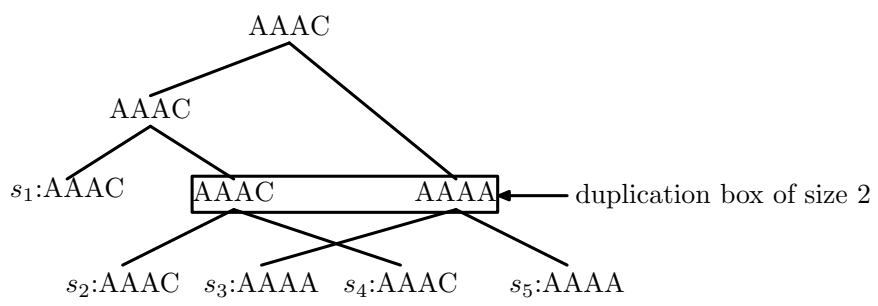

Figure 2: A duplication model with cost 1 for a string $S=s_{1} s_{2} s_{3} s_{4} s_{5}$ of five length-4 segments, where $s_{1}=A A A C, s_{2}=A A A C, s_{3}=A A A A, s_{4}=A A A C$, and $s_{5}=A A A A$.

If we represent only the parent-child relations defined by a duplication model $M$, then the resulting structure $T_{M}$ is planar and can be drawn without edge crossings. $T_{M}$ is a rooted binary tree for the given string $S=s_{1} s_{2} \ldots s_{n}$ of $n$ segments and is called the associated phylogeny for $M$. Obviously, if every block in $M$ is of size 1 , then $T_{M}$ and $M$ are identical. However, if one or more blocks in $M$ are of size larger than 1, then the left-to-right ordering of the labels assigned to the leaves of $T_{M}$ is not $s_{1}, s_{2}, \ldots, s_{n}$.

\subsection{The Problem and the Results}

Now, we are ready to state the problem considered in the paper:

\section{Duplication History Reconstruction (DHR):}

- Input: An ordered list $\left\langle s_{1}, s_{2}, \ldots, s_{n}\right\rangle$ of strings of the same length $m$.

- Output: A duplication model for the string $S=s_{1} s_{2} \ldots s_{n}$ with the smallest cost.

For each integer $k \geq 1$, let $k$-DHR denote the special case of DHR where the size of each duplication block is at most $k$. In this paper, we consider only 1-DHR and 2-DHR. Our first result is a PTAS for 1-DHR; it achieves a ratio of $1+\frac{2^{h}}{h 2^{h}-2 q}$ in $O\left(n^{\ell+1}+m n^{\ell}\right)$ time, where $h=\left\lceil\log _{2} \ell\right\rceil$ and $q=2^{h}-\ell$. Our PTAS is faster than the previously best PTAS for 1-DHR given in [4]. For example, to achieve a ratio of 1.5 , our PTAS takes $O\left(n^{5}+m n^{4}\right)$ time while the PTAS in [4] takes $O\left(n^{11}+m n^{5}\right)$ time.

Our second result is a polynomial-time approximation algorithm for 2-DHR that achieves a ratio of 6 . This is the first polynomial-time approximation algorithm with a guaranteed ratio for 2-DHR. Our algorithm is based on a hidden structure, called the component tree, of a duplication model. 


\section{The PTAS for 1-DHR}

A semiphylogeny is a rooted tree $T$ satisfying the following conditions (see Figure $3(\mathrm{a})$ for an example):

- Each vertex of $T$ is assigned a string and all the strings assigned to the vertices of $T$ have the same length.

- Each internal vertex $v$ of $T$ may be unifurcate or bifurcate. In the former case, $v$ has only one child. In the latter case, $v$ has two children.

- The root of $T$ is bifurcate.

- Both the parent and the child of each unifurcate internal vertex of $T$ are bifurcate.

- The children of each bifurcate internal vertex $v$ of $T$ are distinguished as the left child and the right child of $v$.

- For every unifurcate internal vertex $v$ in $T$ that is the left child of its parent in $T$, the string assigned to $v$ in $T$ is the same as the string assigned to $r(v, T)$, where $r(v, T)$ is the rightmost descendant of $v$ in $T$. (Comment: $r(v, T)$ must be a leaf in $T$ because the child of each unifurcate vertex is bifurcate.)

- For every unifurcate internal vertex $v$ in $T$ that is the right child of its parent in $T$, the string assigned to $v$ in $T$ is the same as the string assigned to $l(v, T)$, where $l(v, T)$ is the leftmost descendant of $v$ in $T$. (Comment: $l(v, T)$ must be a leaf in $T$ because the child of each unifurcate vertex is bifurcate.)

A semiphylogeny is a phylogeny if it has no unifurcate internal vertices.

Let $T$ be a semiphylogeny. An edge $e$ of $T$ is artificial if $e$ connects a unifurcate internal vertex of $T$ to its (unique) child in $T$; otherwise, it is natural. If we delete all artificial edges from $T$, then we get a forest in which each connected component is a phylogeny; we use $\mathcal{D}(T)$ to denote the set of these phylogenies. Note that the number of phylogenies in $\mathcal{D}(T)$ is 1 plus the number of artificial edges in $T$. We call each phylogeny in $\mathcal{D}(T)$ a subphylogeny of $T$.

For an integer $\ell \geq 2$, an $\ell$-semiphylogeny is a semiphylogeny $T$ such that each subphylogeny of $T$ has at most $\ell$ leaves. The cost of a semiphylogeny $T$, denoted by $c(T)$, is the total cost of edges in its subphylogenies. In other words, $c(T)$ is the total cost of natural edges in $T$.

A semiphylogeny for an (ordered) list $\left\langle s_{1}, \ldots, s_{n}\right\rangle$ of strings of the same length is a semiphylogeny whose leaves are assigned the strings $s_{1}, \ldots, s_{n}$ from left to right in this order. Note that 1-DHR

can be restated as the problem of constructing a phylogeny for a given list of strings of the same length.

Lemma 2.1 A semiphylogeny $T$ for a list $\left\langle s_{1}, \ldots, s_{n}\right\rangle$ of strings of the same length can be easily transformed into a phylogeny $T^{\prime}$ for the same list with $c\left(T^{\prime}\right)=c(T)$.

Proof. We assemble the phylogenies in $\mathcal{D}(T)$ into a single phylogeny $T^{\prime}$ for $\left\langle s_{1}, \ldots, s_{n}\right\rangle$ as follows (see Figure 3): 


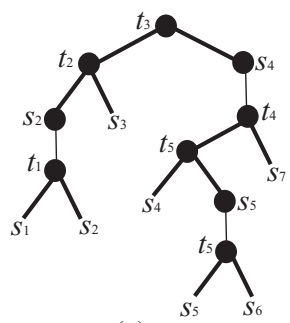

(a)

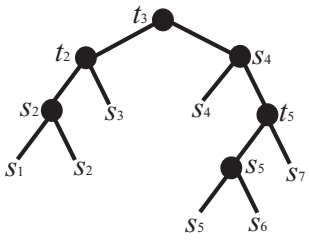

(b)

Figure 3: (a) A 3-semiphylogeny $T$ where the bold edges are the natural edges. (b) The phylogeny $T^{\prime}$ constructed from $T$.

1. Initialize $T^{\prime}$ as the forest consisting of the trees in $\mathcal{D}(T)$.

2. For each unifurcate internal vertex $v$ of $T$ that is the left child of its parent in $T$, add the edge $(v, r(v, T))$ to $T^{\prime}$, connect a new vertex $v^{\prime}$ to $r(v, T)$ by a new edge, and assign $v^{\prime}$ the string that is assigned to $r(v, T)$ in $T$.

3. For each unifurcate internal vertex $v$ of $T$ that is the right child of its parent in $T$, add the edge $(v, l(v, T))$ to $T^{\prime}$, connect a new vertex $v^{\prime}$ to $l(v, T)$ by a new edge, and assign $v^{\prime}$ the string that is assigned to $l(v, T)$ in $T$. (Comment: After this step, $T^{\prime}$ is a tree in which each new vertex is a leaf.)

4. Root $T^{\prime}$ at $r$, where $r$ is the root of $T$.

5. Order the children of each internal vertex in $T^{\prime}$ accordingly so that the strings $s_{1}, \ldots, s_{n}$ appear at the leaves of $T^{\prime}$ from left to right in this order.

6. While $T^{\prime}$ has a unifurcate internal vertex, select such a vertex and merge the two edges incident to it into a single edge.

The main idea behind our PTAS for 1-DHR is that for any given constant $\ell$, we can construct a minimum-cost $\ell$-semiphylogeny for a given list of strings of the same length in polynomial time via dynamic programming. The details will be given in Section 2.1.

Now, our PTAS for 1-DHR is as follows:

Input: An ordered list $\left\langle s_{1}, \ldots, s_{n}\right\rangle$ of strings of the same length and an integer $\ell \geq 2$.

1. Compute a minimum-cost $\ell$-semiphylogeny $T$ for $\left\langle s_{1}, \ldots, s_{n}\right\rangle$.

2. Transform $T$ into a phylogeny $T^{\prime}$ for $\left\langle s_{1}, \ldots, s_{n}\right\rangle$ as described in Lemma 2.1.

Output: $T^{\prime}$.

In order to prove that the above algorithm is indeed a PTAS for 1-DHR, we also need to show that the minimum cost of an $\ell$-semiphylogeny for a list of strings is very close to the minimum cost of a phylogeny for the same list of strings. Sections 2.2 through 2.4 are for this purpose. 


\subsection{Algorithm for Optimal $\ell$-Semiphylogenies}

Throughout this subsection, let $\left\langle s_{1}, \ldots, s_{n}\right\rangle$ be a given list of two or more strings of the same length $m$, and let $\ell$ be an integer larger than 1 . The goal is to construct a minimum-cost $\ell$-semiphylogeny for $\left\langle s_{1}, \ldots, s_{n}\right\rangle$ via dynamic programming.

First, for each ordered list $L$ of strings among $s_{1}, \ldots, s_{n}$ with $2 \leq|L| \leq \ell$ and for each unlabeled binary tree $U$ with $|L|$ leaves, we compute a minimum-cost phylogeny $T_{L, U}$ for $L$ such that the topology of $T_{L, U}$ is the same as $U$. Given $L$ and $U, T_{L, U}$ can be computed in $O\left(m|L|^{3}\right)$ time by a simple dynamic programming. Moreover, for each integer $k \geq 2$, there are exactly $C_{k-1}$ unlabeled binary trees with $k$ leaves, where $C_{k-1}=\frac{(2 k-2) !}{k !(k-1) !}$ is known as the $(k-1)$-th Catalan number. It is also widely known that $C_{k-1}$ is approximately $\frac{4^{k-1}}{\sqrt{\pi}(k-1)^{1.5}}$. Thus, the total time needed for computing all $T_{L, U}$ is $O\left(m n^{\ell}\right)$ time.

For two integers $i$ and $j$ with $1 \leq i \leq j \leq n$, let $c_{i, j}$ be the cost of a minimum-cost $\ell$ semiphylogeny $T_{i, j}$ for $\left\langle s_{i}, \ldots, s_{j}\right\rangle$. Consider the computation of $c_{i, j}$ with $j-i+1>\ell$. Imagine that we already have $T_{i, j}$ and consider $\mathcal{D}\left(T_{i, j}\right)$. One phylogeny $T_{\text {top }}$ in $\mathcal{D}\left(T_{i, j}\right)$ contains the root of $T_{i, j}$. Let $k$ be the number of leaves in $T_{\text {top }}$. Note that $2 \leq k \leq \ell$.

Let $v_{1}, \ldots, v_{k}$ be the leaves of $T_{t o p}$ (ordered from left to right). Note that some $v_{h}$ with $1 \leq h \leq k$ may also be a leaf of $T_{i, j}$. For each $h \in\{1, \ldots, k\}$, let $T_{i, j}\left(v_{h}\right)$ denote the subtree of $T_{i, j}$ rooted at $v_{h}$ (i.e., the subtree of $T_{i, j}$ formed by $v_{h}$ and its descendants in $T_{i, j}$ ). Note that if $v_{h}$ is a leaf of $T_{i, j}$, then $T_{i, j}\left(v_{h}\right)$ has only one vertex (namely, $v_{h}$ ) and hence this vertex is both the root and the (unique) leaf of $T_{i, j}\left(v_{h}\right)$. For each $h \in\{1, \ldots, k\}$, let $s_{a_{h}}, s_{a_{h}+1}, \ldots, s_{b_{h}}$ be the strings assigned to the leaves of $T_{i, j}\left(v_{h}\right)$ (from left to right) in $T_{i, j}$. Obviously, $a_{1}=i, b_{k}=j$, and $a_{h+1}=b_{h}+1$ for each $h \in\{1, \ldots, k-1\}$. Moreover, for each $v_{h} \in\left\{v_{1}, \ldots, v_{k}\right\}$, if $v_{h}$ is a leaf of $T_{i, j}$, then $a_{h}=b_{h}$; otherwise, the following hold:

- If $v_{h}$ is the left child of its parent in $T_{t o p}$, then the string assigned to $v_{h}$ is $s_{b_{h}}$.

- If $v_{h}$ is the right child of its parent in $T_{t o p}$, then the string assigned to $v_{h}$ is $s_{a_{h}}$.

We also have $c\left(T_{i, j}\right)=c\left(T_{\text {top }}\right)+\sum_{h=1}^{k} c\left(T_{i, j}\left(v_{h}\right)\right)=c\left(T_{L, U}\right)+\sum_{h=1}^{k} c\left(T_{i, j}\left(v_{h}\right)\right)$, where $L$ is the list of strings assigned to the leaves of $T_{\text {top }}$ and $U$ is the topology of $T_{\text {opt }}$.

Therefore, when $j-i+1>\ell$, we have the following equation:

$$
c_{i, j}=\min _{2 \leq k \leq \ell} \min _{i \leq b_{1}<b_{2}<\ldots<b_{k-1}<j} \min _{U}\left\{c\left(T_{L, U}\right)+\sum_{h=1}^{k} c_{b_{h-1}+1, b_{h}}\right\},
$$

where $b_{0}=i-1, b_{k}=j, U$ ranges over all unlabeled binary trees with exactly $k$ leaves, and $L$ is the ordered list of $k$ strings defined as follows:

- For each $h \in\{1, \ldots, k\}$ such that $b_{h}-b_{h-1}=1$, the $h$-th string in $L$ is $s_{b_{h}}$.

- For each $h \in\{1, \ldots, k\}$ such that $b_{h}-b_{h-1} \geq 2$, if the $h$-th leftmost leaf of $U$ is the left child of its parent in $U$, then the $h$-th string in $L$ is $s_{b_{h}}$; otherwise, the $h$-th string in $L$ is $s_{b_{h-1}+1}$. 
Recall that there are $O\left(\frac{4^{k}}{k}\right)$ choices for $U$ in Equation (2.1). Given $U$ and $L, T_{U, L}$ is already available. So, $c_{i, j}$ can be computed in $O\left(4^{\ell}(j-i+1)^{\ell-1} \ell\right)$ time. Obviously, there are $O\left(n^{2}\right)$ pairs $(i, j)$. Thus, $c_{1, n}$ can be computed in $O\left(n^{\ell+1}+m n^{\ell}\right)$ time.

In summary, we have proven:

Theorem 2.2 For every integer $\ell \geq 2$, we can compute a minimum-cost $\ell$-semiphylogeny for a given list of $n$ length-m strings in $O\left(n^{\ell+1}+m n^{\ell}\right)$ time.

\subsection{Transforming Phylogenies into $\ell$-Semiphylogenies}

To show that the minimum cost of an $\ell$-semiphylogeny for a list $\left\langle s_{1}, \ldots, s_{n}\right\rangle$ of strings of the same length is very close to the minimum cost of a phylogeny for $\left\langle s_{1}, \ldots, s_{n}\right\rangle$, our idea is to show that a minimum-cost phylogeny for $\left\langle s_{1}, \ldots, s_{n}\right\rangle$ can be transformed into an $\ell$-semiphylogeny for $\left\langle s_{1}, \ldots, s_{n}\right\rangle$ without increasing its cost significantly.

Let $T_{o p t}$ be a minimum-cost phylogeny for $\left\langle s_{1}, \ldots, s_{n}\right\rangle$. For a path $P$ in $T_{\text {opt }}$, we use $c(P)$ to denote the total cost of edges in $P$. Moreover, we use $I\left(T_{o p t}\right)$ to denote the set of all internal vertices of $T_{\text {opt }}$ other than the root.

For each $v \in I\left(T_{o p t}\right)$, we define a path $P(v)$ as follows: If $v$ is the left child of its parent in $T_{o p t}$, then $P(v)$ is the path in $T_{o p t}$ from $v$ to $r\left(v, T_{o p t}\right)$; otherwise, $P(v)$ is the path in $T_{o p t}$ from $v$ to $l\left(v, T_{\text {opt }}\right)$ (see Figure 4.)

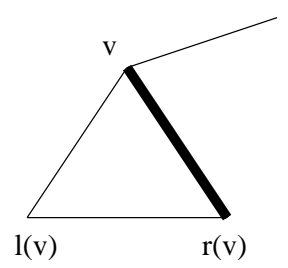

(a)

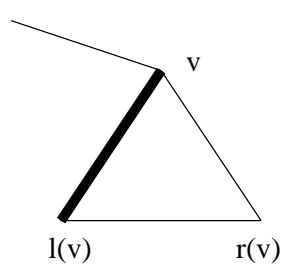

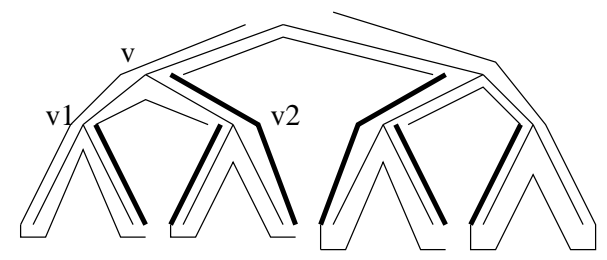

(b)

Figure 4: (a) $P(v)$ in two cases. (b) A concrete example.

Lemma 2.3 $\sum_{v \in I\left(T_{o p t}\right)} c(P(v)) \leq c\left(T_{o p t}\right)$.

Proof. To prove the lemma, it suffices to claim that for two distinct vertices $u$ and $v$ in $I\left(T_{o p t}\right)$, $P(u)$ and $P(v)$ are edge-disjoint. The claim is not obvious only when one of $u$ and $v$ is an ancestor of the other in $T_{o p t}$. So, assume that $u$ is an ancestor of $v$ in $T_{o p t}$. Then, $P(u)$ and $P(v)$ are clearly edge-disjoint if $v$ is not a vertex of $P(u)$. So, let us further assume that $v$ is a vertex of $P(u)$. Then, when $u$ is the left child of its parent in $T_{o p t}, v$ must be the right child of its parent in $T_{o p t}$ and hence $P(v)$ is on the left of $P(u)$. Similarly, when $u$ is the right child of its parent in $T_{\text {opt }}, v$ must be the left child of its parent in $T_{\text {opt }}$ and hence $P(v)$ is on the right of $P(u)$. Thus, in both cases, $P(u)$ and $P(v)$ are edge-disjoint.

Since the Hamming distance is a metric, we also have:

Lemma 2.4 For every $v \in I\left(T_{\text {out }}\right)$, the Hamming distance between the strings assigned to $r\left(v, T_{\text {opt }}\right)$ (respectively, $l\left(v, T_{\text {opt }}\right)$ ) and the parent $u$ of $v$ in $T_{\text {opt }}$ is at most $c(P(v))$ plus the cost of the edge between $v$ and $u$ in $T_{\text {opt }}$. 
For a rooted tree $T$, we define the level of each vertex $v$ in $T$ (denoted by $l e v(v, T)$ ) as follows: The root is at level 0 and its children are at level 1 . In general, if a vertex is at level $i \geq 0$, then its children are at level $i+1$.

Fix an integer $\ell \geq 2$. Our goal is to transform $T_{\text {opt }}$ into an $\ell$-semiphylogeny $T$ for $s_{1}, \ldots, s_{n}$ such that $c(T)$ is very close to $c\left(T_{\text {opt }}\right)$. To begin, we let $T$ be a copy of $T_{\text {opt }}$. Then, we find a subset $J$ of $I\left(T_{o p t}\right)$ (in some way to be specified later in Sections 2.3 and 2.4) and modify $T$ by repeating the following five steps until $J$ becomes empty:

1. Find a vertex $v \in J$ whose level in $T_{o p t}$ is the minimum among all vertices of $J$.

2. Split the edge $e_{v}$ of $T$ between $v$ and its parent $v_{p}$ in $T$ into two edges by adding a new vertex $v^{\prime}$ at the middle of $e_{v}$.

3. If $v^{\prime}$ is the left child of $v_{p}$ in $T$, then assign $v^{\prime}$ the string that is assigned to $r\left(v, T_{\text {opt }}\right)$ in $T_{\text {opt }}$. (Comment: After this step, $v^{\prime}$ is a unifurcate internal vertex of semiphylogeny $T$ and the cost of the (natural) edge between $v_{p}$ and $v^{\prime}$ in $T$ is at most $c(P(v))$ plus the cost of $e_{v}$ according to Lemma 2.4. So, this step increases the cost of $T$ by at most $c(P(v))$.)

4. If $v^{\prime}$ is the right child of $v_{p}$ in $T$, then assign $v^{\prime}$ the string that is assigned to $l\left(v, T_{\text {opt }}\right)$ in $T_{\text {opt }}$. (Comment: Same as the comment on the last step.)

5. Remove $v$ from $J$.

We use $T_{\text {opt }}(J)$ to denote the semiphylogeny $T$ obtained from $T_{\text {opt }}$ and $J$ as above. Then, we have:

Lemma 2.5 For every subset $J$ of $I\left(T_{\text {opt }}\right), c\left(T_{\text {opt }}(J)\right) \leq c\left(T_{\text {opt }}\right)+\sum_{v \in J} c(P(v))$.

Unfortunately, depending on $J, T_{\text {opt }}(J)$ may not be an $\ell$-semiphylogeny. So, we say that a subset $J$ of $I\left(T_{\text {opt }}\right)$ is $\ell$-proper if $T_{\text {opt }}(J)$ is an $\ell$-semiphylogeny. What remains to show is that for every integer $\ell \geq 2$, there is always an $\ell$-proper subset of $I\left(T_{o p t}\right)$ such that $\sum_{v \in J} c(P(v))$ is relatively small compared to $c\left(T_{\text {opt }}\right)$. The next two subsections are for this purpose.

\subsection{The Ratio when $\ell$ Is a Power of 2}

This case is easy as shown in the next lemma:

Lemma 2.6 For every integer $\ell=2^{h}$ with $h \geq 1$, there is always an $\ell$-proper subset $J$ of $I\left(T_{\text {opt }}\right)$ such that $\sum_{v \in J} c(P(v)) \leq \frac{1}{h} \cdot c\left(T_{\text {opt }}\right)$.

Proof. Let $r$ be the root of $T_{\text {opt }}$. For each integer $j$ with $0 \leq j \leq h-1$, let $V_{j}$ be the set of those vertices $v \in I\left(T_{\text {opt }}\right)$ such that $l e v\left(v, T_{\text {opt }}\right) \equiv j(\bmod h)$. Obviously, $V_{j}$ is $\ell$-proper. Moreover, $V_{j} \cap V_{j^{\prime}}=\emptyset$ for $0 \leq j \neq j^{\prime} \leq h-1$. Hence,

$$
\sum_{j=0}^{h-1} \sum_{v \in V_{j}} c(P(v)) \leq \sum_{v \in I\left(T_{o p t}\right)} c(P(v)) \leq c\left(T_{o p t}\right),
$$


where the last inequality follows from Lemma 2.3. Consequently, there exists an integer $j \in$ $\{0,1, \ldots, h-1\}$ such that $\sum_{v \in V_{j}} c(P(v)) \leq \frac{1}{h} \cdot c\left(T_{o p t}\right)$.

Combining Theorem 2.2 and Lemmas 2.1 and 2.6, we now have:

Theorem 2.7 Our algorithm is a PTAS for 1 -DHR and achieves a ratio of $1+\frac{1}{h}$ in $O\left(n^{2^{h}+1}+\right.$ $m n^{2^{h}}$ ) time for every integer $h \geq 1$.

For example, to achieve a ratio of 1.5 , our PTAS takes $O\left(n^{5}+m n^{4}\right)$ time while the PTAS in [4] takes $O\left(n^{11}+m n^{5}\right)$ time.

\subsection{The Ratio When $\ell$ Is not a Power of 2}

This case is much more difficult. We start by giving several definitions. The height of a binary tree is the maximum level of a vertex in the tree. A binary tree is complete if it has $2^{k}$ leaves, where $k$ is the height of the tree. Note that for each integer $k \geq 0$, there is a unique unlabeled complete binary tree of height $k$. Moreover, in a complete binary tree, all leaves appear at the same level. A binary tree $T$ is balanced if every internal vertex of $T$ has two children in $T$ and the level of every leaf in $T$ is $k$ or $k-1$, where $k$ is the height of $T$.

Since $\ell$ is not a power of $2, \ell \geq 3$ and $\ell$ can be written as $\ell=2^{h}-q$, where $h=\left\lceil\log _{2} \ell\right\rceil$ and $q=2^{h}-\ell$. Obviously, from a complete binary tree $T$ of height $k$, we can obtain a balanced binary tree of height $h$ and with $\ell$ leaves by choosing $q$ vertices at the $(h-1)$-th level in $T$ and deleting their children from $T$. So, there are only $b=\left(\begin{array}{c}2^{h-1} \\ q\end{array}\right)$ unlabeled balanced binary trees of height $h$ and with $\ell$ leaves. We denote them by $B_{1}, \ldots, B_{b}$ (see Figures 5 and 6 for two examples).

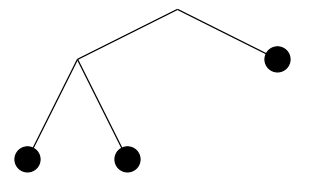

(a)

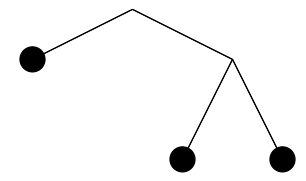

(b)

Figure 5: $B_{1}$ and $B_{2}$ when $\ell=3$.

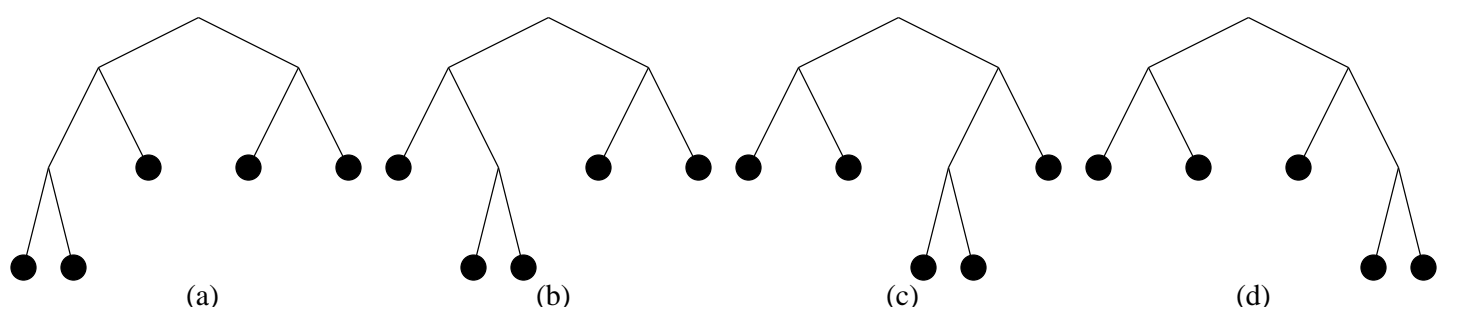

Figure 6: $B_{1}, \ldots, B_{4}$ when $\ell=5$.

We choose a tree $B_{a}$ among $B_{1}, \ldots, B_{b}$ uniformaly at random. For each $i \in\{0, \ldots, h-1\}$, we will use $B_{a}$ to find an $\ell$-proper subset $J_{i}$ of $I\left(T_{o p t}\right)$. The description of $J_{i}$ will be much easier if $T_{o p t}$ is a complete binary tree. So, we repeat the following steps until $T_{\text {opt }}$ becomes a complete binary tree:

1. Select a leaf $v$ whose level in $T_{o p t}$ is smaller than the height of $T_{o p t}$. 
2. Introduce two new vertices $v^{\prime}$ and $v^{\prime \prime}$ and connect each of them to $v$ by an edge.

3. Assign each of the new leaves $v^{\prime}$ and $v^{\prime \prime}$ the string that is assigned to $v$.

Obviously, $T_{o p t}$ is still a phylogeny but may not be a phylogeny for $\left\langle s_{1}, \ldots, s_{n}\right\rangle$ because it may have more than $n$ leaves. However, the cost and the height of $T_{\text {opt }}$ remain the same. For convenience, we denote the original $T_{\text {opt }}$ by $T_{\text {opt }}^{\text {org }}$.

We say that a vertex $v$ of $T_{\text {opt }}$ is sufficiently low in $T_{\text {opt }}$ if $l e v\left(v, T_{\text {opt }}\right) \leq h_{\text {opt }}-h$, where $h_{\text {opt }}$ is the height of $T_{\text {opt }}$. For each sufficiently low vertex $v$ of $T_{o p t}$, we define a subtree $T_{\text {opt }}\left(v, B_{a}\right)$ of $T_{o p t}$ as follows:

- The root of $T_{o p t}\left(v, B_{a}\right)$ is $v$.

- $T_{\text {opt }}\left(v, B_{a}\right)$ is isomorphic to $B_{a}$, i.e., there is a one-to-one mapping $\varphi$ from the vertices of $T_{\text {opt }}\left(v, B_{a}\right)$ to the vertices of $B_{a}$ such that

$-\varphi(v)$ is the root of $B_{a}$ and

- for every vertex $u$ of $T_{o p t}\left(v, B_{a}\right)$ other than $v$, if $u$ is the left (respectively, right) child of its parent $u^{\prime}$ in $T_{\text {opt }}\left(v, B_{a}\right)$, then $\varphi(u)$ is the left (respectively, right) child of $\varphi\left(u^{\prime}\right)$ in $B_{a}$.

Note that $T_{\text {opt }}\left(v, B_{a}\right)$ must exist because $v$ is sufficiently low and $T_{\text {opt }}$ is a complete binary tree.

We are now ready to use $B_{a}$ to find an $\ell$-proper subset $J_{i}$ of $I\left(T_{\text {opt }}\right)$ for each $i \in\{0, \ldots, h-1\}$ as follows:

1. Initialize $J_{i}$ to be the set of all vertices at level $i$ in $T_{o p t}$.

2. For each vertex $v$ of $T_{\text {opt }}$, if $v \in J_{i}$ and $v$ is sufficiently low in $T_{\text {opt }}$, then color $v$ black; otherwise, color $v$ white.

3. While $T_{\text {opt }}$ has a black vertex, perform the following steps:

(a) Select an arbitrary black vertex $v$ in $T_{o p t}$ and recolor $v$ white in $T_{o p t}$.

(b) Add all leaves of $T_{o p t}\left(v, B_{a}\right)$ to $J_{i}$.

(c) Color those leaves of $T_{o p t}\left(v, B_{a}\right)$ in $T_{o p t}$ black that are also sufficiently low in $T_{o p t}$.

Note that $J_{0}$ contains the root $r$ of $T_{\text {opt }}$ and we delete it from $J_{0}$. Then, for each $i \in\{0, \ldots, h-1\}$, $J_{i}$ is an $\ell$-proper subset of $I\left(T_{\text {opt }}\right)$.

Lemma 2.8 For each $i \in\{0, \ldots, h-1\}$, let $J_{i}^{\prime}=J_{i} \cap I\left(T_{\text {opt }}^{\text {org }}\right)$. Then, the following statements hold:

1. For each $i \in\{0, \ldots, h-1\}, J_{i}^{\prime}$ is an $\ell$-proper subset of $I\left(T_{\text {opt }}^{\text {org }}\right)$ in $T_{\text {opt }}^{\text {org }}$.

2. If $\alpha$ is a real number (depending on $\ell$ ) such that $\sum_{v \in J_{i}} c(P(v)) \leq \alpha \cdot c\left(T_{o p t}\right)$, then $\sum_{v \in J_{i}^{\prime}} c(P(v)) \leq$ $\alpha \cdot c\left(T_{\text {opt }}^{\text {org }}\right)$. 
Proof. The first statement follows from the fact that $T_{\text {opt }}^{\text {org }}$ is a subtree of $T_{\text {opt }}$. The second statement follows from the fact that $c(P(v))=0$ for every $v \in I\left(T_{\text {opt }}\right)-I\left(T_{\text {opt }}^{\text {org }}\right)$.

By Lemma 2.8, what remains to do is to prove that there is a small number (depending on $\ell$ ) such that $\sum_{v \in J_{i}} c(P(v)) \leq \alpha \cdot c\left(T_{o p t}\right)$. The proof is not easy because two sets among $J_{0}, \ldots, J_{h-1}$ may not be disjoint. We give the proof below.

For each $i \in\{0, \ldots, h-1\}$ and for each $j \in\left\{0, \ldots, h_{\text {opt }}\right\}$, let $y_{i}(j)$ be the expected number of vertices $v \in J_{i}$ with $\operatorname{lev}\left(v, T_{o p t}\right)=j$. By the construction of each $J_{i}$, we have the following lemma immediately:

Lemma 2.9 The following statements hold:

1. $y_{0}(0)=\cdots=y_{0}(h-2)=0, y_{0}(h-1)=q, y_{0}(h)=2^{h}-2 q$, and

$$
y_{0}(j)=q \cdot y_{0}(j-h+1)+\left(2^{h}-2 q\right) \cdot y_{0}(j-h) \text { for all } j \geq h+1 \text {. }
$$

2. For every $i \in\{1, \ldots, h-1\}, y_{i}(0)=\cdots=y_{i}(i-1)=0, y_{i}(i)=2^{i}, y_{i}(i+1)=\cdots=$ $y_{i}(i+h-2)=0, y_{i}(i+h-1)=2^{i} q$, and

$$
y_{i}(j)=q \cdot y_{i}(j-h+1)+\left(2^{h}-2 q\right) \cdot y_{i}(j-h) \text { for all } j \geq i+h .
$$

For each $i \in\{0, \ldots, h-1\}$ and for each $j \in\left\{0, \ldots, h_{\text {opt }}\right\}$, we define

$$
x_{i}(j)=\frac{y_{i}(j)}{2^{j}} .
$$

Since $B_{a}$ is chosen from $B_{1}, \ldots, B_{b}$ uniformly at random, each vertex at level $j$ in $T_{\text {opt }}$ has the same probability to be included in $J_{i}$. So, for each vertex $v$ with $l e v\left(v, T_{o p t}\right)=j$,

$$
x_{i}(j)=\operatorname{Pr}\left[v \in J_{i}\right], \text { which is the probability that } v \in J_{i} .
$$

Moreover, by Lemma 2.9, we have the following lemma immediately:

Lemma 2.10 The following statements hold:

1. $x_{0}(0)=\cdots=x_{0}(h-2)=0, x_{0}(h-1)=\frac{q}{2^{h-1}}, x_{0}(h)=\frac{2^{h}-2 q}{2^{h}}$, and

$$
x_{0}(j)=\frac{2 q}{2^{h}} \cdot x_{0}(j-h+1)+\frac{2^{h}-2 q}{2^{h}} \cdot x_{0}(j-h) \text { for all } j \geq h+1 .
$$

2. For every $i \in\{1, \ldots, h-1\}, x_{i}(0)=\cdots=x_{i}(i-1)=0, x_{i}(i)=1, x_{i}(i+1)=\cdots=$ $x_{i}(i+h-2)=0, x_{i}(i+h-1)=\frac{q}{2^{h-1}}$, and

$$
x_{i}(j)=\frac{2 q}{2^{h}} \cdot x_{i}(j-h+1)+\frac{2^{h}-2 q}{2^{h}} \cdot x_{i}(j-h) \text { for all } j \geq i+h .
$$

3. For each $i \in\{1, \ldots, h-1\}$ and for each $j \in\left\{1, \ldots, h_{\text {opt }}\right\}, x_{i}(j)=x_{i-1}(j-1)$, except that $1=x_{1}(1) \neq x_{0}(0)=0$. 
Note that Statement 3 in Lemma 2.10 follows from Statements 1 and 2 in the same lemma.

It seems very difficult to solve the recurrence relations in Lemma 2.10. We get around the difficulty by considering the following weighted sum of $x_{0}(j), \ldots, x_{h-1}(j)$ for all $j \geq 0$ :

$$
X(j)=2^{h} \sum_{i=0}^{h-2} x_{i}(j)+\left(2^{h}-2 q\right) \cdot x_{h-1}(j) .
$$

Lemma 2.11 $X(0)=0$ and $X(j)=2^{h}$ for all $j \geq 1$.

Proof. Using Statements 1 and 2 in Lemma 2.10, it is easy to verify that $X(0)=0$ and $X(1)=\cdots=X(h)=2^{h}$. So, consider an arbitrary integer $j>h$. By Statement 3 in Lemma 2.10,

$$
X(j)=2^{h} \sum_{i=0}^{h-2} x_{0}(j-i)+\left(2^{h}-2 q\right) \cdot x_{0}(j-h+1) .
$$

Thus,

$$
X(j)-X(j-1)=2^{h} \cdot x_{0}(j)-2 q \cdot x_{0}(j-h+1)-\left(2^{h}-2 q\right) \cdot x_{0}(j-h) .
$$

Hence, by the recurrence relation in Statement 1 in Lemma 2.10, $X(j)-X(j-1)=0$. Therefore, $X(j)=X(j-1)=\cdots=X(h)=2^{h}$.

Next, we consider $\mathcal{E}\left[\sum_{v \in J_{i}} c(P(v))\right]$, which is the expected value of $\sum_{v \in J_{i}} c(P(v))$. For each $j \geq 0$, let $t(j)=\sum_{v} c(P(v))$, where $v$ ranges over all vertices $v$ of $T_{\text {opt }}$ with $l e v\left(v, T_{\text {opt }}\right)=j$. Then, we have:

$$
\mathcal{E}\left[\sum_{v \in J_{i}} c(P(v))\right]=\sum_{j=1}^{h_{\text {opt }}} x_{i}(j) \cdot t(j)
$$

because $\operatorname{Pr}\left[v \in J_{i}\right]=x_{i}(j)$ for each vertex $v$ of $T_{o p t}$ with $\operatorname{lev}\left(v, T_{o p t}\right)=j$.

Now, we turn to the following weighted sum of $\mathcal{E}\left[\sum_{v \in J_{0}} c(P(v))\right], \ldots, \mathcal{E}\left[\sum_{v \in J_{h-1}} c(P(v))\right]$ :

$$
W=2^{h} \cdot \mathcal{E}\left[\sum_{v \in J_{0}} c(P(v))\right]+\cdots+2^{h} \cdot \mathcal{E}\left[\sum_{v \in J_{h-2}} c(P(v))\right]+\left(2^{h}-2 q\right) \cdot \mathcal{E}\left[\sum_{v \in J_{h-1}} c(P(v))\right],
$$

which can be rewritten as

$$
2^{h} \cdot \sum_{j=1}^{h_{\text {opt }}} x_{0}(j) \cdot t(j)+\cdots+2^{h} \cdot \sum_{j=1}^{h_{\text {opt }}} x_{h-2}(j) \cdot t(j)+\left(2^{h}-2 q\right) \cdot \sum_{j=1}^{h_{\text {opt }}} x_{h-1}(j) \cdot t(j)
$$

and further as

$$
\sum_{j=1}^{h_{\text {opt }}} t(j) \cdot\left(2^{h} \cdot x_{0}(j)+\cdots+2^{h} \cdot x_{h-2}(j)+\left(2^{h}-2 q\right) \cdot x_{h-1}(j)\right) .
$$

So, we have

$$
W=\sum_{j=1}^{h_{o p t}} t(j) \cdot X(j)=2^{h} \sum_{j=1}^{h_{\text {opt }}} t(j) \leq 2^{h} \cdot c\left(T_{\text {opt }}\right),
$$

where the last inequality follows from Lemma 2.3. Thus, the weighted average of $\mathcal{E}\left[\sum_{v \in J_{0}} c(P(v))\right]$, $\ldots, \mathcal{E}\left[\sum_{v \in J_{h-1}} c(P(v))\right]$ is

$$
\frac{1}{2^{h} h-2 q} \cdot W \leq \frac{2^{h}}{2^{h} h-2 q} \cdot c\left(T_{\text {opt }}\right)
$$


Hence, there is an $i \in\{0, \ldots, h-1\}$ such that

$$
\mathcal{E}\left[\sum_{v \in J_{i}} c(P(v))\right] \leq \frac{2^{h}}{2^{h} h-2 q} \cdot c\left(T_{o p t}\right) .
$$

Therefore, there is a choice of $B_{a}$ from $\left\{B_{1}, \ldots, B_{b}\right\}$ such that the subset $J_{i}$ constructed from $B_{a}$ as above satisfies the following inequality:

$$
\sum_{v \in J_{i}} c(P(v)) \leq \frac{2^{h}}{2^{h} h-2 q} \cdot c\left(T_{o p t}\right) .
$$

In summary, we have proven the following lemma:

Lemma 2.12 For every integer $\ell \geq 3$ (that is not a power of 2), there is always an $\ell$-proper subset $J$ of $I\left(T_{\text {opt }}\right)$ such that $\sum_{v \in J} c(P(v)) \leq \frac{2^{h}}{2^{h} h-2 q} \cdot c\left(T_{\text {opt }}\right)$, where $h=\left\lceil\log _{2} \ell\right\rceil$ and $q=2^{h}-\ell$.

Combining Theorem 2.2 and Lemmas 2.1 and 2.12, we now have:

Theorem 2.13 Our algorithm is a PTAS for 1 -DHR and achieves a ratio of $1+\frac{2^{h}}{2^{h} h-2 q}$ in $O\left(n^{\ell+1}+\right.$ $m n^{\ell}$ ) time for every integer $\ell \geq 3$ (that is not a power of 2), where $h=\left\lceil\log _{2} \ell\right\rceil$ and $q=2^{h}-\ell$.

For example, to achieve a ratio of $\frac{5}{3}$, our PTAS takes $O\left(n^{4}+m n^{3}\right)$ time while the PTAS in [4] takes $O\left(n^{7}+m n^{3}\right)$ time.

\section{A Ratio-6 Approximation Algorithm for 2-DHR}

Throughout this section, fix a list $\left\langle s_{1}, \ldots, s_{n}\right\rangle$ of strings of the same length $m$, and let $M_{\text {opt }}$ be a minimum-cost duplication model for $s_{1} s_{2} \ldots s_{n}$.

One problem with $M_{o p t}$ is that the label assigned to an internal vertex in $M_{o p t}$ may not be in $\left\{s_{1}, \ldots, s_{n}\right\}$. This makes it hard to compute $M_{o p t}$. So instead, we look for a restricted type of duplication models called lifted duplication models. In a lifted duplication model, the label assigned to each internal vertex is a string in $\left\{s_{1}, \ldots, s_{n}\right\}$. The following lemma shows that there is always a good lifted duplication model:

Lemma 3.1 There is a lifted duplication model $N_{\text {opt }}$ for $s_{1} s_{2} \ldots s_{n}$ such that $c\left(N_{\text {opt }}\right) \leq 2 \cdot c\left(M_{\text {opt }}\right)$.

Proof. Recall that $T_{M_{\text {opt }}}$ denotes the associated phylogeny for $M_{\text {opt }}$. Note that $c\left(M_{\text {opt }}\right)=$ $c\left(T_{M_{\text {opt }}}\right)$. Using the lifting technique in [10], we can elaborately lift the labels of the leaves in $T_{M_{\text {opt }}}$ up to their ancestors so that the resulting phylogeny $T$ has a cost less than or equal to $2 \cdot c\left(T_{M_{o p t}}\right)$. Now, since the vertices of $M_{o p t}$ one-to-one correspond to those of $T$, we can obtain a new model $N_{\text {opt }}$ from $M_{\text {opt }}$ by simply changing the label of each vertex in $M_{o p t}$ to that of the corresponding vertex in $T$. Obviously, $N_{\text {opt }}$ is a lifted duplication model for $s_{1} s_{2} \ldots s_{n}$. Moreover, $c\left(N_{\text {opt }}\right)=c(T) \leq 2 \cdot c\left(T_{M_{\text {opt }}}\right)=2 \cdot c\left(M_{\text {opt }}\right)$.

The rest of this section is organized as follows. In Section 3.1, we give several definitions and prove several lemmas. In Section 3.2, we define the component tree $\mathcal{D}(M)$ of a given lifted duplication model $M$ for $s_{1} s_{2} \ldots s_{n}$. In Section 3.3, we show how to construct a new lifted duplication model $M^{\prime}$ from $\mathcal{D}(M)$. The crucial point is that we can show $c\left(M^{\prime}\right) \leq 3 \cdot c(M)$. In Section 3.4, we describe our algorithm for computing a component tree $\mathcal{D}_{\text {opt }}$ such that the cost of the new lifted duplication model constructed from $\mathcal{D}_{\text {opt }}$ is minimized. 


\subsection{Preliminaries}

Let $M$ be a lifted duplication model for $s_{1} s_{2} \ldots s_{n}$. An edge in $M$ is planar if it is not crossed by another edge in $M$. A path in $M$ is planar if it traverses planar edges only. Two internal vertices $u$ and $v$ in $M$ are incomparable if $u$ is neither an ancestor nor a descendant of $v$ in $M$.

Each size-2 block $B$ in $M$ contains two internal vertices; we distinguish them as the left vertex and the right vertex in $B$. For each block $B$, we define the leftmost planar path of $B$ and the rightmost planar path of $B$ as follows: If the size of $B$ is 1 , then both paths start at the unique internal vertex in $B$; otherwise, the leftmost planar path of $B$ starts at the left vertex in $B$ and the rightmost planar path of $B$ starts at the right vertex in $B$. The leftmost (respectively, rightmost) planar path of $B$ then goes down all the way to a leaf by only traversing planar edges based on the following rule:

- When going down from an internal vertex $u$ to one of its children, always choose the left (respectively, right) child $v$ of $u$ in $B$ if the edge $(u, v)$ is planar. (Comment: Since $M$ is a duplication model, at least one of the two edges between $u$ and its children is planar. So, the path can always go down to a leaf by traversing planar edges only.)

An extreme planar path in $M$ is a path that is the leftmost or rightmost of some block in $M$. The next lemma is obvious but helps the reader understand the relations between extreme planar paths in $M$.

Lemma 3.2 The following statements hold:

1. If an internal vertex $v$ of $M$ is contained in a size-1 block, then there are exactly two extreme planar paths starting at $v$ and $v$ is the unique vertex shared by the two paths.

2. If an internal vertex $v$ of $M$ is contained in a size-2 block, then there is exactly one extreme planar path starting at $v$.

3. If $u$ and $v$ are two incomparable internal vertices in $M$, then no extreme planar path starting at $u$ shares a vertex with an extreme planar path starting at $v$.

For each integer $i$ with $1 \leq i \leq n$, the $i$-th leftmost leaf in $M$ is labeled with the string $s_{i}$. For convenience, we also use $s_{i}$ to denote the $i$-th leftmost leaf in $M$. For each internal vertex $u$ of $M$, we use $a(u)$ (respectively, $b(u)$ ) to denote the integer $i$ such that $s_{i}$ is the leftmost (respectively, rightmost) leaf descendant of $u$ in $M$. Two internal vertices $u$ and $v$ are unrelated in $M$ if $b(u)<a(v)$ or $b(v)<a(u)$. An internal vertex $u$ crosses another internal vertex $v$ in $M$ if $a(u)<a(v)<b(u)<b(v)$. An internal vertex $u$ covers another internal vertex $v$ in $M$ if $a(u) \leq a(v)<b(v) \leq b(u)$. Note that if $u$ is ancestor of $v$ in $M$, then $u$ covers $v$ in $M$. However, an internal vertex may cover another internal vertex in $M$ even if they are incomparable in $M$. Two internal vertices of $M$ are unnested if no one of them covers the other in $M$.

The next lemma is obvious but helps the reader understand how a vertex crosses another vertex in $M$.

Lemma 3.3 If a vertex $u$ crosses another vertex $v$ in $M$, then the path $P_{u}$ from $u$ to $s_{b(u)}$ in $M$ and the path $P_{v}$ from $v$ to $s_{a(v)}$ in $M$ cross each other exactly once and hence there is exactly one size-2 box $B_{u, v}$ in $M$ whose left vertex is on $P_{u}$ and whose right vertex is on $P_{v}$. 
We call the block $B_{u, v}$ in Lemma 3.3 the block for $(u, v)$-crossing in $M$.

The next two lemmas help the reader understand the relations between unnested internal vertices in $M$.

Lemma 3.4 There do not exist three pairwise unnested internal vertices $u, v$, and $w$ in $M$ such that $u$ crosses $v$ in $M$ and so does $w$.

Proof. Suppose that an internal vertex $u$ crosses another internal vertex $v$ in $M$. Let $\left\langle x_{1}, y_{1}\right\rangle$ be the block for $(u, v)$-crossing in $M$. Suppose that an internal vertex $w$ other than $u$ also crosses $v$ in $M$. Let $\left\langle x_{2}, y_{2}\right\rangle$ be the block for $(w, v)$-crossing in $M$. Then, both $y_{1}$ and $y_{2}$ appear on the path $P_{v}$ from $v$ to $s_{a(v)}$ in $M$. So, one of $y_{1}$ and $y_{2}$ is an ancestor of the other in $M$. Without loss of generality, we may assume that $y_{1}$ is an ancestor of $y_{2}$ in $M$. Then, the leftmost planar path starting at $x_{1}$ must end at a leaf $s_{i}$ with $i<a(w)$. So, $a(u)<i<a(w)$ because $u$ covers $x_{1}$ in $M$. Similarly, the rightmost planar path starting at $y_{2}$ must end at a leaf $s_{k}$ with $b(w)<k<b(u)$. Thus, $u$ covers $w$ in $M$. This establishes the lemma.

Lemma 3.5 Suppose that $u, v$, and $w$ are three pairwise unnested internal vertices in $M$ such that $u$ crosses $v$ in $M$ and $v$ crosses $w$ in $M$. Then, $a(v)<b(u)<a(w)<b(v)$ and hence $u$ does not cross $w$ in $M$.

Proof. Since $u$ crosses $v$ in $M$, we have $a(v)<b(u)$ immediately. Similarly, $a(w)<b(v)$. To prove $b(u)<a(w)$, consider the right vertex $x$ in the block for $(u, v)$-crossing and the left vertex $y$ in the block for $(v, w)$-crossing. Obviously, the rightmost planar path $P_{x}$ starting at $x$ must end at a leaf $s_{i}$ with $b(u)<i$ and the leftmost planar path $P_{y}$ starting at $y$ must end at a leaf $s_{j}$ with $j<a(w)$. Moreover, since $x$ is on the left of $y$ in $M, P_{x}$ is on the left of $P_{y}$ and so $i<j$. Hence, $b(u)<i<j<a(w)$.

A triple $(L, i, j)$ is closed in $M$ if either $i=j$ and $L$ consists of $s_{i}$ only, or the following conditions are satisfied:

- $i$ and $j$ are integers with $1 \leq i<j \leq n$.

- $L$ is an ordered list $\left\langle v_{1}, v_{2}, \ldots, v_{k}\right\rangle$ of pairwise unnested internal vertices with $1 \leq k \leq 4$.

- The left child of $v_{1}$ in $M$ is an ancestor of $s_{i}$, and the right child of $v_{k}$ in $M$ is an ancestor of $s_{j}$.

- The path from $v_{1}$ to $s_{i}$ in $M$ is planar and so is the path from $v_{k}$ to $s_{j}$ in $M$.

- For each integer $h$ with $2 \leq h \leq k-1$, all leaf descendants of $v_{h}$ in $M$ are among $s_{i}, s_{i+1}$, $\ldots, s_{j}$.

- Every leaf $s_{\ell}$ with $i \leq \ell \leq j$ is a descendant of some $v_{h}$ with $1 \leq h \leq k$ in $M$.

- For each integer $h$ with $1 \leq h \leq k-1, v_{h}$ crosses $v_{h+1}$ in $M$. 
For example, both $\left(\left\langle t_{2}\right\rangle, 1,4\right)$ and $\left(\left\langle t_{5}, t_{6}\right\rangle, 4,15\right)$ are closed triples in the duplication model in Figure 7(a).

For each closed triple $(L, i, j)$ in $M$, we use $M(L, i, j)$ to denote the subgraph of $M$ induced by the set of those vertices $u$ in $M$ such that $u$ is a vertex in $L, u$ is a leaf $s_{h}$ with $i \leq h \leq j$, or $u$ is both an ancestor of some $s_{h}$ with $i \leq h \leq j$ and a descendant of some vertex of $L$ in $M$. For example, $M(\langle r\rangle, 1, n)$ is exactly $M$, where $r$ is the root of $M$.

Note that $M(L, i, j)$ is a forest consisting of $|L|$ rooted trees whose roots are the vertices in $L$ and whose leaves are $s_{i}, \ldots, s_{j}$. Moreover, $M(L, i, j)$ may have unifurcate vertices, which are those vertices having only one child in $M(L, i, j)$. Obviously, each unifurcate vertex in $M(L, i, j)$ appears on the path from the leaf $s_{i}$ to the first vertex in $L$ or on the path from the leaf $s_{j}$ to the last vertex in $L$.

For each closed triple $(L, i, j)$ in $M$, we call $M(L, i, j)$ a component of $M$ and call the vertices in $L$ the roots of the component. Note that the roots of $M(L, i, j)$ are ordered from left to right. For example, the leftmost root is the first root while the rightmost root is the last root.

\subsection{The Component Tree of a Model}

We inherit the notations in Section 3.1. For each vertex $v$ of $M$, let $s(v)$ denote the string assigned to $v$ in $M$. Moreover, for a list $L$ of vertices in $M$, let $s(L)$ denote the list of strings assigned to the vertices in $L$. Furthermore, for two strings $s^{\prime}$ and $s^{\prime \prime}$, let $d\left(s^{\prime}, s^{\prime \prime}\right)$ denote the hamming distance between them.

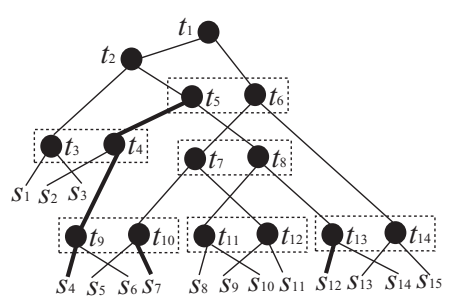

(a)

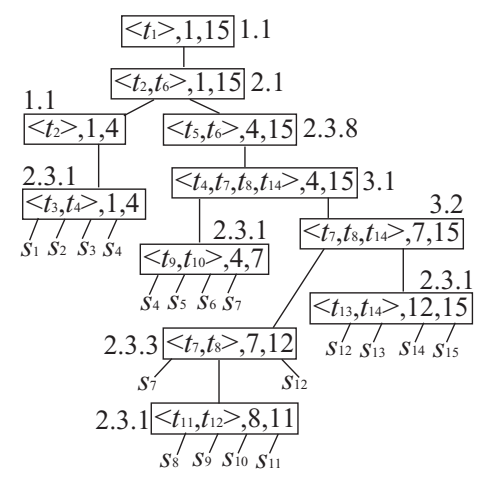

(b)

Figure 7: (a) A duplication model $M$ where the three bold paths are the splitting paths for obtaining $\mathcal{D}(M)$. (b) The component tree $\mathcal{D}(M)$ of $M$ where the type of each node is given near the node, the weight of each edge is omitted, and the label $\left(\left\langle s_{i}\right\rangle, i, i\right)$ of each leaf is simplified to $s_{i}$.

We want to dissect $M$ into components and organize them into a rooted, ordered, edge-weighted, and node-labeled tree $\mathcal{D}(M)$, called the component tree of $M$ (see Figure 7 for an example). Since we want to do this recursively, it is more convenient to dissect $M(L, i, j)$ into components and organize them into a rooted, ordered, edge-weighted, and node-labeled tree $\mathcal{D}_{M}(L, i, j)$ (called the component tree of $M(L, i, j))$ for each closed triple $(L, i, j)$ in $M$.

We next construct $\mathcal{D}_{M}(L, i, j)$ by induction on $j-i$. In the base case where $j-i=0, \mathcal{D}_{M}(L, i, j)$ has only one node $\alpha(L, i, j)$; we label the node with $(s(L), i, j)$ and call it a type- 0 node. So, suppose that $j-i \geq 1$. Then, depending on $|L|$, there are three cases. In each case, we first create a root 
node $\alpha(L, i, j)$ for $\mathcal{D}_{M}(L, i, j)$ and label it with $(s(L), i, j)$. Then, we proceed to grow $\mathcal{D}_{M}(L, i, j)$ in each case as follows:

Case 1: $|L|=1$. Let $u$ be the root of $M(L, i, j)$, and let $v_{1}$ (respectively, $v_{2}$ ) be the first vertex on the path from $u$ to the leaf $s_{i}$ (respectively, $s_{j}$ ) that is not a unifurcate internal vertex in $M(L, i, j)$. We further distinguish two subcases as follows:

Case 1.1: $v_{1}$ crosses $v_{2}$ in $M$. In this subcase, $\left(\left\langle v_{1}, v_{2}\right\rangle, i, j\right)$ is a closed triple in $M$. So, we recursively construct $\mathcal{D}_{M}\left(\left\langle v_{1}, v_{2}\right\rangle, i, j\right)$, then let $\alpha\left(\left\langle v_{1}, v_{2}\right\rangle, i, j\right)$ be the unique child of $\alpha(L, i, j)$, and further let the weight of the edge between $\alpha(L, i, j)$ and its child be $d\left(s(u), s\left(v_{1}\right)\right)+d\left(s(u), s\left(v_{2}\right)\right)$. We also call $\alpha(L, i, j)$ a type-1.1 node.

Case 1.2: $v_{1}$ does not cross $v_{2}$ in $M$. In this subcase, both $\left(\left\langle v_{1}\right\rangle, i, b\left(v_{1}\right)\right)$ and $\left(\left\langle v_{2}\right\rangle, a\left(v_{2}\right), j\right)$ are closed triples in $M$. So, we recursively construct $\mathcal{D}_{M}\left(\left\langle v_{1}\right\rangle, i, b\left(v_{1}\right)\right)$ and $\mathcal{D}_{M}\left(\left\langle v_{2}\right\rangle, a\left(v_{2}\right), j\right)$. We then let $\alpha\left(\left\langle v_{1}\right\rangle, i, b\left(v_{1}\right)\right)$ and $\alpha\left(\left\langle v_{2}\right\rangle, a\left(v_{2}\right), j\right)$ be the left and the right child of $\alpha(L, i, j)$, respectively. We further let the weight of the edge between $\alpha(L, i, j)$ and its left (respectively, right) child be $d\left(s(u), s\left(v_{1}\right)\right)$ (respectively, $d\left(s(u), s\left(v_{2}\right)\right)$ ). We also call $\alpha(L, i, j)$ a type-1.2 node.

Case 2: $|L|=2$. Let $u_{1}$ and $u_{2}$ be the roots of $M(L, i, j)$. Let $\left\langle v_{1}, v_{2}\right\rangle$ be the block for $\left(u_{1}, u_{2}\right)$-crossing in $M$. We further distinguish three subcases as follows:

Case 2.1: $u_{1} \neq v_{1}$. Let $s_{k}$ be the leaf at which the leftmost planar path starting at $v_{1}$ ends. Then, $\left(\left\langle u_{1}\right\rangle, i, k\right)$ and $\left(\left\langle v_{1}, u_{2}\right\rangle, k, j\right)$ are closed triples in $M$. So, we recursively construct $\mathcal{D}_{M}\left(\left\langle u_{1}\right\rangle, i, k\right)$ and $\mathcal{D}_{M}\left(\left\langle v_{1}, u_{2}\right\rangle, k, j\right)$. Intuitively speaking, this means that we split $M(L, i, j)$ into two components along the path from $v_{1}$ to $s_{k}$ and then construct their component trees recursively. For convenience, we call the path a splitting path and associate it with $\alpha(L, i, j)$. Now, to finish constructing $\mathcal{D}_{M}(L, i, j)$, we let $\alpha\left(\left\langle u_{1}\right\rangle, i, k\right)$ and $\alpha\left(\left\langle v_{1}, u_{2}\right\rangle, k, j\right)$ be the left and the second child of $\alpha(L, i, j)$, respectively. We further let the weight of the edge between $\alpha(L, i, j)$ and each of its children be $\frac{1}{2} d\left(s_{k}, s\left(v_{1}\right)\right)$. We also call $\alpha(L, i, j)$ a type-2.1 node.

Case 2.2: $u_{1}=v_{1}$ but $u_{2} \neq v_{2}$. Let $s_{\ell}$ be the leaf at which the rightmost planar path starting at $v_{2}$ ends. Then, $\left(\left\langle u_{1}, v_{2}\right\rangle, i, \ell\right)$ and $\left(\left\langle u_{2}\right\rangle, \ell, j\right)$ are closed triples in $M$. So, we recursively construct $\mathcal{D}_{M}\left(\left\langle u_{1}, v_{2}\right\rangle, i, \ell\right)$ and $\mathcal{D}_{M}\left(\left\langle u_{2}\right\rangle, \ell, j\right)$. Intuitively speaking, this means that we split $M(L, i, j)$ into two components along the path from $v_{2}$ to $s_{\ell}$ and then construct their component trees recursively. For convenience, we call the path a splitting path and associate it with $\alpha(L, i, j)$. Now, to finish constructing $\mathcal{D}_{M}(L, i, j)$, we let $\alpha\left(\left\langle u_{1}, v_{2}\right\rangle, i, \ell\right)$ and $\alpha\left(\left\langle u_{2}\right\rangle, \ell, j\right)$ be the left and the right child of $\alpha(L, i, j)$, respectively. We further let the weight of the edge between $\alpha(L, i, j)$ and each of its children be $\frac{1}{2} d\left(s_{\ell}, s\left(v_{2}\right)\right)$. We also call $\alpha(L, i, j)$ a type-2.2 node.

Case 2.3: $u_{1}=v_{1}$ and $u_{2}=v_{2}$. Let $w_{1}$ (respectively, $w_{4}$ ) be the first vertex on the path from $u_{1}$ (respectively, $u_{2}$ ) to the leaf $s_{i}$ (respectively, $s_{j}$ ) that is not a unifurcate internal vertex in $M(L, i, j)$. Let $w_{3}$ (respectively, $w_{2}$ ) be the right (respectively, left) child of $u_{1}$ (respectively, $u_{2}$ ) in $M(L, i, j)$. By Lemma 3.5, one of the following subsubcases occurs:

Case 2.3.1: There do not exist two vertices $w_{i}$ and $w_{j}$ with $1 \leq i<j \leq 4$ such that $w_{i}$ crosses $w_{j}$ in $M$. Obviously, $\left(\left\langle w_{1}\right\rangle, i, b\left(w_{1}\right)\right),\left(\left\langle w_{2}\right\rangle, a\left(w_{2}\right), b\left(w_{2}\right)\right),\left(\left\langle w_{3}\right\rangle, a\left(w_{3}\right), b\left(w_{3}\right)\right)$, and $\left(\left\langle w_{4}\right\rangle, a\left(w_{4}\right), j\right)$ are closed triples in $M$. Note that $a\left(w_{h}\right)=b\left(w_{h-1}\right)+1$ for $2 \leq h \leq 4$. So, we recursively construct $\mathcal{D}_{M}\left(\left\langle w_{1}\right\rangle, i, b\left(w_{1}\right)\right), \mathcal{D}_{M}\left(\left\langle w_{2}\right\rangle, a\left(w_{2}\right), b\left(w_{2}\right)\right), \mathcal{D}_{M}\left(\left\langle w_{3}\right\rangle, a\left(w_{3}\right), b\left(w_{3}\right)\right)$, and $\mathcal{D}_{M}\left(\left\langle w_{4}\right\rangle, a\left(w_{4}\right), j\right)$. We then let $\alpha\left(\left\langle w_{1}\right\rangle, i, b\left(w_{1}\right)\right), \alpha\left(\left\langle w_{2}\right\rangle, a\left(w_{2}\right), b\left(w_{2}\right)\right), \alpha\left(\left\langle w_{3}\right\rangle, a\left(w_{3}\right), b\left(w_{3}\right)\right)$, and $\alpha\left(\left\langle w_{4}\right\rangle, a\left(w_{4}\right), j\right)$ be the first (i.e., the leftmost), the second, the third, and the fourth child of $\alpha(L, i, j)$, respectively. We further let the weight of the edge between $\alpha(L, i, j)$ and its first, its second, its third, and its fourth 
child be $d\left(s\left(u_{1}\right), s\left(w_{1}\right)\right), d\left(s\left(u_{2}\right), s\left(w_{2}\right)\right), d\left(s\left(u_{1}\right), s\left(w_{3}\right)\right)$, and $d\left(s\left(u_{2}\right), s\left(w_{4}\right)\right)$, respectively. We also call $\alpha(L, i, j)$ a type-2.3.1 node.

Case 2.3.2: $w_{1}$ crosses $w_{2}$ in $M$ but neither $w_{2}$ crosses $w_{3}$ nor $w_{3}$ crosses $w_{4}$ in $M$. Obviously, $\left(\left\langle w_{1}, w_{2}\right\rangle, i, b\left(w_{2}\right)\right),\left(\left\langle w_{3}\right\rangle, a\left(w_{3}\right), b\left(w_{3}\right)\right)$, and $\left(\left\langle w_{4}\right\rangle, a\left(w_{4}\right), j\right)$ are closed triples in $M$. Note that $a\left(w_{3}\right)=b\left(w_{2}\right)+1$ and $a\left(w_{4}\right)=b\left(w_{3}\right)+1$. So, we recursively construct $\mathcal{D}_{M}\left(\left\langle w_{1}, w_{2}\right\rangle, i, b\left(w_{2}\right)\right)$, $\mathcal{D}_{M}\left(\left\langle w_{3}\right\rangle, a\left(w_{3}\right), b\left(w_{3}\right)\right)$, and $\mathcal{D}_{M}\left(\left\langle w_{4}\right\rangle, a\left(w_{4}\right), j\right)$. We then let $\alpha\left(\left\langle w_{1}, w_{2}\right\rangle, i, b\left(w_{2}\right)\right), \alpha\left(\left\langle w_{3}\right\rangle, a\left(w_{3}\right), b\left(w_{3}\right)\right)$, and $\alpha\left(\left\langle w_{4}\right\rangle, a\left(w_{4}\right), j\right)$ be the left, the middle, and the right child of $\alpha(L, i, j)$, respectively. We further let the weight of the edge between $\alpha(L, i, j)$ and its left, its middle, and its right child be $d\left(s\left(u_{1}\right), s\left(w_{1}\right)\right)+d\left(s\left(u_{2}\right), s\left(w_{2}\right)\right), d\left(s\left(u_{1}\right), s\left(w_{3}\right)\right)$, and $d\left(s\left(u_{2}\right), s\left(w_{4}\right)\right)$, respectively. We also call $\alpha(L, i, j)$ a type-2.3.2 node.

Case 2.3.3: $w_{2}$ crosses $w_{3}$ in $M$ but neither $w_{1}$ crosses $w_{2}$ nor $w_{3}$ crosses $w_{4}$ in $M$. Obviously, $\left(\left\langle w_{1}\right\rangle, i, b\left(w_{1}\right)\right),\left(\left\langle w_{2}, w_{3}\right\rangle, a\left(w_{2}\right), b\left(w_{3}\right)\right)$, and $\left(\left\langle w_{4}\right\rangle, a\left(w_{4}\right), j\right)$ are closed triples in $M$. Note that $a\left(w_{2}\right)=b\left(w_{1}\right)+1$ and $a\left(w_{4}\right)=b\left(w_{3}\right)+1$. So, we recursively construct $\mathcal{D}_{M}\left(\left\langle w_{1}\right\rangle, i, b\left(w_{1}\right)\right)$, $\mathcal{D}_{M}\left(\left\langle w_{2}, w_{3}\right\rangle, a\left(w_{2}\right), b\left(w_{3}\right)\right)$, and $\mathcal{D}_{M}\left(\left\langle w_{4}\right\rangle, a\left(w_{4}\right), j\right)$. We then let $\alpha\left(\left\langle w_{1}\right\rangle, i, b\left(w_{1}\right)\right), \alpha\left(\left\langle w_{2}, w_{3}\right\rangle, a\left(w_{2}\right), b\left(w_{3}\right)\right)$, and $\alpha\left(\left\langle w_{4}\right\rangle, a\left(w_{4}\right), j\right)$ be the left, the middle, and the right child of $\alpha(L, i, j)$, respectively. We further let the weight of the edge between $\alpha(L, i, j)$ and its left, its middle, and its right child be $d\left(s\left(u_{1}\right), s\left(w_{1}\right)\right), d\left(s\left(u_{1}\right), s\left(w_{3}\right)\right)+d\left(s\left(u_{2}\right), s\left(w_{2}\right)\right)$, and $d\left(s\left(u_{2}\right), s\left(w_{4}\right)\right)$, respectively. We also call $\alpha(L, i, j)$ a type-2.3.3 node.

Case 2.3.4: $w_{3}$ crosses $w_{4}$ in $M$ but neither $w_{1}$ crosses $w_{2}$ nor $w_{2}$ crosses $w_{3}$ in $M$. Obviously, $\left(\left\langle w_{1}\right\rangle, i, b\left(w_{1}\right)\right),\left(\left\langle w_{2}\right\rangle, a\left(w_{2}\right), b\left(w_{2}\right)\right)$, and $\left(\left\langle w_{3}, w_{4}\right\rangle, a\left(w_{3}\right), j\right)$ are closed triples in $M$. Note that $a\left(w_{2}\right)=b\left(w_{1}\right)+1$ and $a\left(w_{3}\right)=b\left(w_{2}\right)+1$. So, we recursively construct $\mathcal{D}_{M}\left(\left\langle w_{1}\right\rangle, i, b\left(w_{1}\right)\right)$, $\mathcal{D}_{M}\left(\left\langle w_{2}\right\rangle, a\left(w_{2}\right), b\left(w_{2}\right)\right)$, and $\mathcal{D}_{M}\left(\left\langle w_{3}, w_{4}\right\rangle, a\left(w_{3}\right), j\right)$. We then let $\alpha\left(\left\langle w_{1}\right\rangle, i, b\left(w_{1}\right)\right), \alpha\left(\left\langle w_{2}\right\rangle, a\left(w_{2}\right), b\left(w_{2}\right)\right)$, and $\alpha\left(\left\langle w_{3}, w_{4}\right\rangle, a\left(w_{3}\right), j\right)$ be the left, the middle, and the right child of $\alpha(L, i, j)$, respectively. We further let the weight of the edge between $\alpha(L, i, j)$ and its left, its middle, and its right child be $d\left(s\left(u_{1}\right), s\left(w_{1}\right)\right), d\left(s\left(u_{2}\right), s\left(w_{2}\right)\right)$, and $d\left(s\left(u_{1}\right), s\left(w_{3}\right)\right)+d\left(s\left(u_{2}\right), s\left(w_{4}\right)\right)$, respectively. We also call $\alpha(L, i, j)$ a type-2.3.4 node.

Case 2.3.5: Both $w_{1}$ crosses $w_{2}$ and $w_{2}$ crosses $w_{3}$ but $w_{3}$ does not cross $w_{4}$ in $M$. Obviously, $\left(\left\langle w_{1}, w_{2}, w_{3}\right\rangle, i, b\left(w_{3}\right)\right)$ and $\left(\left\langle w_{4}\right\rangle, a\left(w_{4}\right), j\right)$ are closed triples in $M$. Note that $a\left(w_{4}\right)=b\left(w_{3}\right)+$ 1. So, we recursively construct $\mathcal{D}_{M}\left(\left\langle w_{1}, w_{2}, w_{3}\right\rangle, i, b\left(w_{3}\right)\right)$ and $\mathcal{D}_{M}\left(\left\langle w_{4}\right\rangle, a\left(w_{4}\right), j\right)$. We then let $\alpha\left(\left\langle w_{1}, w_{2}, w_{3}\right\rangle, i, b\left(w_{3}\right)\right)$ and $\alpha\left(\left\langle w_{4}\right\rangle, a\left(w_{4}\right), j\right)$ be the left and the right child of $\alpha(L, i, j)$, respectively. We further let the weight of the edge between $\alpha(L, i, j)$ and its left (respectively, right) child be $d\left(s\left(u_{1}\right), s\left(w_{1}\right)\right)+d\left(s\left(u_{2}\right), s\left(w_{2}\right)\right)+d\left(s\left(u_{1}\right), s\left(w_{3}\right)\right)$ (respectively, $d\left(s\left(u_{2}\right), s\left(w_{4}\right)\right)$ ). We also call $\alpha(L, i, j)$ a type-2.3.5 node.

Case 2.3.6: Both $w_{1}$ crosses $w_{2}$ and $w_{3}$ crosses $w_{4}$ but $w_{2}$ does not cross $w_{3}$ in $M$. Obviously, $\left(\left\langle w_{1}, w_{2}\right\rangle, i, b\left(w_{2}\right)\right)$ and $\left(\left\langle w_{3}, w_{4}\right\rangle, a\left(w_{3}\right), j\right)$ are closed triples in $M$. Note that $a\left(w_{3}\right)=b\left(w_{2}\right)+$ 1. So, we recursively construct $\mathcal{D}_{M}\left(\left\langle w_{1}, w_{2}\right\rangle, i, b\left(w_{2}\right)\right)$ and $\mathcal{D}_{M}\left(\left\langle w_{3}, w_{4}\right\rangle, a\left(w_{3}\right), j\right)$. We then let $\alpha\left(\left\langle w_{1}, w_{2}\right\rangle, i, b\left(w_{2}\right)\right)$ and $\alpha\left(\left\langle w_{3}, w_{4}\right\rangle, a\left(w_{3}\right), j\right)$ be the left and the right child of $\alpha(L, i, j)$, respectively. We further let the weight of the edge between $\alpha(L, i, j)$ and its left (respectively, right) child be $d\left(s\left(u_{1}\right), s\left(w_{1}\right)\right)+d\left(s\left(u_{2}\right), s\left(w_{2}\right)\right)$ (respectively, $\left.d\left(s\left(u_{1}\right), s\left(w_{3}\right)\right)+d\left(s\left(u_{2}\right), s\left(w_{4}\right)\right)\right)$. We also call $\alpha(L, i, j)$ a type-2.3.6 node.

Case 2.3.7: Both $w_{2}$ crosses $w_{3}$ and $w_{3}$ crosses $w_{4}$ but $w_{1}$ does not cross $w_{2}$ in $M$. Obviously, $\left(\left\langle w_{1}\right\rangle, i, b\left(w_{1}\right)\right)$ and $\left(\left\langle w_{2}, w_{3}, w_{4}\right\rangle, a\left(w_{2}\right), j\right)$ are closed triples in $M$. Note that $a\left(w_{2}\right)=b\left(w_{1}\right)+$ 1. So, we recursively construct $\mathcal{D}_{M}\left(\left\langle w_{1}\right\rangle, i, b\left(w_{1}\right)\right)$ and $\mathcal{D}_{M}\left(\left\langle w_{2}, w_{3}, w_{4}\right\rangle, a\left(w_{2}\right), j\right)$. We then let 
$\alpha\left(\left\langle w_{1}\right\rangle, i, b\left(w_{1}\right)\right)$ and $\alpha\left(\left\langle w_{2}, w_{3}, w_{4}\right\rangle, a\left(w_{2}\right), j\right)$ be the left and the right child of $\alpha(L, i, j)$, respectively. We further let the weight of the edge between $\alpha(L, i, j)$ and its left (respectively, right) child be $d\left(s\left(u_{1}\right), s\left(w_{1}\right)\right)$ (respectively, $\left.d\left(s\left(u_{2}\right), s\left(w_{2}\right)\right)+d\left(s\left(u_{1}\right), s\left(w_{3}\right)\right)+d\left(s\left(u_{2}\right), s\left(w_{4}\right)\right)\right)$. We also call $\alpha(L, i, j)$ a type-2.3.7 node.

Case 2.3.8: For each integer $h$ with $1 \leq h \leq 3, w_{h}$ crosses $w_{h+1}$ in $M$. Obviously, $\left(\left\langle w_{1}, w_{2}, w_{3}, w_{4}\right\rangle, i, j\right)$ is a closed triple in $M$. So, we recursively construct $\mathcal{D}_{M}\left(\left\langle w_{1}, w_{2}, w_{3}, w_{4}\right\rangle, i, j\right)$. We then let $\alpha\left(\left\langle w_{1}, w_{2}, w_{3}, w_{4}\right\rangle, i, j\right)$ be the unique child of $\alpha(L, i, j)$. We further let the edge between $\alpha(L, i, j)$ and its child be $d\left(s\left(u_{1}\right), s\left(w_{1}\right)\right)+d\left(s\left(u_{2}\right), s\left(w_{2}\right)\right)+d\left(s\left(u_{1}\right), s\left(w_{3}\right)\right)+d\left(s\left(u_{2}\right), s\left(w_{4}\right)\right)$. We also call $\alpha(L, i, j)$ a type-2.3.8 node.

Case 3: $3 \leq|L| \leq 4$. Let $h=|L|$. Let $u_{1}, \ldots, u_{h}$ be the roots of $M(L, i, j)$. Let $\left\langle v_{1}, v_{2}\right\rangle$ be the block for $\left(u_{1}, u_{2}\right)$-crossing in $M$, and let $\left\langle w_{2}, w_{3}\right\rangle$ be the block for $\left(u_{2}, u_{3}\right)$-crossing in $M$. We further distinguish two subcases as follows:

Case 3.1: $u_{2} \neq v_{2}$. Let $s_{k}$ be the leaf at which the rightmost planar path starting at $v_{2}$ ends. Then, $\left(\left\langle u_{1}, v_{2}\right\rangle, i, k\right)$ and $\left(\left\langle u_{2}, \ldots, u_{h}\right\rangle, k, j\right)$ are closed triples in $M$. So, we recursively construct $\mathcal{D}_{M}\left(\left\langle u_{1}, v_{2}\right\rangle, i, k\right)$ and $\mathcal{D}_{M}\left(\left\langle u_{2}, \ldots, u_{h}\right\rangle, k, j\right)$. Intuitively speaking, this means that we split $M(L, i, j)$ into two components along the path from $v_{2}$ to $s_{k}$ and then construct their component trees recursively. For convenience, we call the path a splitting path and associate it with $\alpha(L, i, j)$. Now, to finish constructing $\mathcal{D}_{M}(L, i, j)$, we just let $\alpha\left(\left\langle u_{1}, v_{2}\right\rangle, i, k\right)$ and $\alpha\left(\left\langle u_{2}, \ldots, u_{h}\right\rangle, k, j\right)$ be the left and the second child of $\alpha(L, i, j)$, respectively. We further let the weight of the edge between $\alpha(L, i, j)$ and each of its child be $\frac{1}{2} d\left(s_{k}, s\left(v_{2}\right)\right)$. We also call $\alpha(L, i, j)$ a type-3.1 node.

Case 3.2: $u_{2}=v_{2}$. Then, $u_{2} \neq w_{2}$. Let $s_{\ell}$ be the leaf at which the leftmost planar path starting at $w_{2}$ ends. Then, $\left(\left\langle u_{1}, u_{2}\right\rangle, i, \ell\right)$ and $\left(\left\langle w_{2}, u_{3}, \ldots, u_{h}\right\rangle, \ell, j\right)$ are closed triples in $M$. So, we recursively construct $\mathcal{D}_{M}\left(\left\langle u_{1}, u_{2}\right\rangle, i, \ell\right)$ and $\mathcal{D}_{M}\left(\left\langle w_{2}, u_{3}, \ldots, u_{h}\right\rangle, \ell, j\right)$. Intuitively speaking, this means that we split $M(L, i, j)$ into two components along the path from $w_{2}$ to $s_{\ell}$ and then construct their component trees recursively. For convenience, we call the path a splitting path and associate it with $\alpha(L, i, j)$. Now, to finish constructing $\mathcal{D}_{M}(L, i, j)$, we just let $\alpha\left(\left\langle u_{1}, u_{2}\right\rangle, i, \ell\right)$ and $\alpha\left(\left\langle w_{2}, u_{3}, \ldots, u_{h}\right\rangle, \ell, j\right)$ be the left and the second child of $\alpha(L, i, j)$, respectively. We further let the edge between $\alpha(L, i, j)$ and each of its child be $\frac{1}{2} d\left(s_{\ell}, s\left(w_{2}\right)\right)$. We also call $\alpha(L, i, j)$ a type-3.2 node.

Lemma 3.6 The component tree $\mathcal{D}(M)$ of $M$ is unique. Moreover, each pair of splitting paths associated with nodes in $\mathcal{D}(M)$ are edge-disjoint.

Proof. The first assertion is obvious from the construction of $\mathcal{D}(M)$. We next prove the second assertion. For each component $M(L, i, j)$ of $M$, we define the left (respectively, right) boundary of $M(L, i, j)$ to be the path from the first (respectively, last) vertex in $L$ to $s_{i}$ (respectively, $s_{j}$ ) in $M$. By examining each case in the above construction of $\mathcal{D}_{M}(L, i, j)$, we can see that if a splitting path is used to split $M(L, i, j)$ into two smaller components, the path does not traverse any edge on the left or right boundary of $M(L, i, j)$ and the splitting path becomes the left or right boundary of each of the smaller components. So, it is impossible for two splitting paths to share an edge.

We define the weight of $\mathcal{D}(M)$ to be the total weight of edges in $\mathcal{D}(M)$.

Lemma 3.7 The weight of $\mathcal{D}(M)$ is at most $3 \cdot c(M)$. 
Proof. For each closed triple $(L, i, j)$, let $c(M(L, i, j))$ be the total cost of edges in $M(L, i, j)$, and let $c\left(\mathcal{D}_{M}(L, i, j)\right)$ be the total weight of edges in $\mathcal{D}_{M}(L, i, j)$. Moreover, if there are one or more splitting paths associated with $\alpha(L, i, j)$ or its descendants in $\mathcal{D}_{M}(L, i, j)$, then let $c_{p}(L, i, j)$ denote the total cost of edges on all the paths; otherwise, let $c_{p}(L, i, j)=0$.

To show the lemma, we first claim that for each closed triple $(L, i, j), c\left(\mathcal{D}_{M}(L, i, j)\right) \leq c(M(L, i, j))+$ $2 \cdot c_{p}(L, i, j)$. We prove the claim by induction on $j-i$. The claim is clearly true when $j-i=0$. So, suppose that $j-i \geq 1$. If no splitting path is associated with $\alpha(L, i, j)$ in $\mathcal{D}_{M}(L, i, j)$, then by the inductive hypothesis and the definition of the weight(s) between $\alpha(L, i, j)$ and its child(ren) in $\mathcal{D}_{M}(L, i, j)$, it is clear that $c\left(\mathcal{D}_{M}(L, i, j)\right) \leq c(M(L, i, j))+2 \cdot c_{p}(L, i, j)$. Otherwise, by the definition of the weights between $\alpha(L, i, j)$ and its children in $\mathcal{D}_{M}(L, i, j)$,

$$
c\left(\mathcal{D}_{M}(L, i, j)\right) \leq c\left(\mathcal{D}_{M}\left(L_{1}, i, k\right)\right)+c\left(\mathcal{D}_{M}\left(L_{2}, k, j\right)\right)+c(P),
$$

where $P$ is the splitting path associated with $\alpha(L, i, j)$ and $\alpha\left(L_{1}, i, k\right)$ and $\left.\alpha\left(L_{2}, k, j\right)\right)$ are the children of $\alpha(L, i, j)$ in $\mathcal{D}_{M}(L, i, j)$. Moreover, we have

$$
\begin{aligned}
& c\left(\mathcal{D}_{M}\left(L_{1}, i, k\right)\right)+c\left(\mathcal{D}_{M}\left(L_{2}, k, j\right)\right)+c(P) \\
\leq & c\left(M\left(L_{1}, i, k\right)\right)+2 \cdot c_{p}\left(L_{1}, i, k\right)+c\left(M\left(L_{2}, k, j\right)\right)+2 \cdot c_{p}\left(L_{2}, k, j\right)+c(P) \\
= & c(M(L, i, j))+2 \cdot c_{p}\left(L_{1}, i, k\right)+2 \cdot c_{p}\left(L_{2}, k, j\right)+2 \cdot c(P) \\
= & c(M(L, i, j))+2 \cdot c_{p}(L, i, j),
\end{aligned}
$$

where the inequality follows from the inductive hypothesis and the first equality follows from the fact that the edges shared by $M\left(L_{1}, i, k\right)$ and $M\left(L_{2}, k, j\right)$ are exactly the edges of $P$. This completes the proof of the claim. The lemma follows from the claim and Lemma 3.6 immediately.

\subsection{Constructing Models from Component Trees}

For convenience, we will allow a duplication model to have unifurcate internal vertices from now on. This does not cause any problem because we can obtain a real duplication model by repeating merging the two edges incident to a unifurcate vertex into a single edge until there are no unifurcate vertices in the model.

We inherit the notations in Sections 3.1 and 3.2. We show how to use $\mathcal{D}(M)$ to construct a duplication model $M^{\prime}$ for $s_{1} s_{2} \ldots s_{n}$ such that $c\left(M^{\prime}\right)$ equals the total weight of edges in $\mathcal{D}(M)$ (see Figure 8 for an example). In the construction of $M^{\prime}$, we will only use the label and the type of each node in $\mathcal{D}(M)$, i.e., we will not look at the topology and the vertices of $M$.

Recall that the label of each node $\beta$ in $\mathcal{D}(M)$ is a triple $(\mathcal{S}, i, j)$, where $\mathcal{S}$ is an ordered nonempty list of at most four (possibly not distinct) strings among $s_{1}, \ldots, s_{n}$ and $i$ and $j$ are two integers with $1 \leq i \leq j \leq n$. For convenience, we call $\mathcal{S}$ the string list of $\beta$.

The construction of $M^{\prime}$ indeed involves constructing a rooted ordered forest $M^{\prime}(\beta)$ for each node $\beta$ of $\mathcal{D}(M)$. We will maintain the invariant that $M^{\prime}(\beta)$ has $|\mathcal{S}|$ roots, where $\mathcal{S}$ is the string list of $\beta$.

We next detail the construction of $M^{\prime}$. We construct $M^{\prime}$ by processing the nodes of $\mathcal{D}(M)$ in a bottom-up fashion. We first process each leaf $\beta$ in $\mathcal{D}(M)$ by constructing $M^{\prime}(\beta)$ as follows: Create a new vertex and assign it the unique string in the string list of $\beta$. 


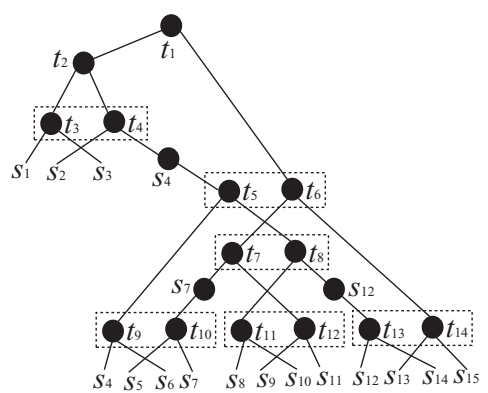

Figure 8: The new duplication model $M^{\prime}$ constructed from the component tree in Figure $7(\mathrm{~b})$.

Now, consider the processing of an internal node $\beta$ in $\mathcal{D}(M)$. Let $\gamma_{1}, \ldots, \gamma_{h}$ be the children of $\beta$ in $\mathcal{D}(M)$, where the ordering is from left to right. Suppose that $M^{\prime}\left(\gamma_{1}\right), \ldots, M^{\prime}\left(\gamma_{h}\right)$ have been constructed by processing $\gamma_{1}, \ldots, \gamma_{h}$. The processing of $\beta$ will depend on its type. Since the possible types of $\beta$ one-to-one correspond to the cases in Section 3.2 , we construct $M^{\prime}(\beta)$ by distinguishing several cases as follows:

Type 1.1 (cf. Case 1.1 in Section 3.2): We create a root vertex for $M^{\prime}(\beta)$, assign it the unique string in the string list of $\beta$, and connect it to the roots of $M^{\prime}\left(\gamma_{1}\right)$ by two new edges. Note that the total cost of the two new edges is exactly the weight of the edge between $\beta$ and $\gamma_{1}$ in $\mathcal{D}(M)$.

Type 1.2 (cf. Case 1.2 in Section 3.2): We create a root vertex $u$ for $M^{\prime}(\beta)$, assign it the unique string in the string list of $\beta$, connect it to the root $v_{1}$ of $M^{\prime}\left(\gamma_{1}\right)$ and to the root $v_{2}$ of $M^{\prime}\left(\gamma_{2}\right)$ so that $v_{1}$ and $v_{2}$ become the left and the right child of $u$ in $M^{\prime}(\beta)$, respectively. Note that the total costs of the new edges $\left(u, v_{1}\right)$ and $\left(u, v_{2}\right)$ in $M^{\prime}(\beta)$ are exactly the total weight of the edges between $\beta$ and its children in $\mathcal{D}(M)$.

Type 2.1 (cf. Case 2.1 in Section 3.2): We connect $M^{\prime}\left(\gamma_{1}\right)$ and $M^{\prime}\left(\gamma_{2}\right)$ by making the left root $v_{2}$ of $M^{\prime}\left(\gamma_{2}\right)$ be the unique child of the rightmost leaf $v_{1}$ of $M^{\prime}\left(\gamma_{1}\right)$. Note that the cost of the new edge $\left(v_{1}, v_{2}\right)$ in $M^{\prime}(\beta)$ is exactly the total weight of the edges between $\beta$ and its children in $\mathcal{D}(M)$.

Type 2.2 (cf. Case 2.2 in Section 3.2): We connect $M^{\prime}\left(\gamma_{1}\right)$ and $M^{\prime}\left(\gamma_{2}\right)$ by making the right root $v_{1}$ of $M^{\prime}\left(\gamma_{1}\right)$ be the unique child of the leftmost leaf $v_{2}$ of $M^{\prime}\left(\gamma_{2}\right)$. Note that the cost of the new edge $\left(v_{2}, v_{1}\right)$ in $M^{\prime}(\beta)$ is exactly the total weight of the edges between $\beta$ and its children in $\mathcal{D}(M)$.

Type 2.3.1 (cf. Case 2.3.1 in Section 3.2): We create two root vertices $u_{1}$ and $u_{2}$ for $M^{\prime}(\beta)$, assign $u_{1}$ the first string in the string list $\mathcal{S}$ of $\beta$, assign $u_{2}$ the second string in $\mathcal{S}$, connect $u_{1}$ to the root $v_{1}$ of $M^{\prime}\left(\gamma_{1}\right)$ and to the root $v_{3}$ of $M^{\prime}\left(\gamma_{3}\right)$ (so that $v_{1}$ and $v_{3}$ become the left and the right child of $u_{1}$ in $M^{\prime}(\beta)$, respectively), and connect $u_{2}$ to the root $v_{2}$ of $M^{\prime}\left(\gamma_{2}\right)$ and to the root $v_{4}$ of $M^{\prime}\left(\gamma_{4}\right)$ (so that $v_{2}$ and $v_{4}$ become the left and the right child of $u_{2}$ in $M^{\prime}(\beta)$, respectively). Note that the total cost of the new edges $\left(u_{1}, v_{1}\right),\left(u_{2}, v_{2}\right),\left(u_{1}, v_{3}\right)$, and $\left(u_{2}, v_{4}\right)$ in $M^{\prime}(\beta)$ is exactly the total weight of the edges between $\beta$ and its children in $\mathcal{D}(M)$.

Type 2.3.2 (cf. Case 2.3.2 in Section 3.2): We create two root vertices $u_{1}$ and $u_{2}$ for $M^{\prime}(\beta)$, assign $u_{1}$ the first string in the string list $\mathcal{S}$ of $\beta$, assign $u_{2}$ the second string in $\mathcal{S}$, connect $u_{1}$ to the left root $v_{1,1}$ of $M^{\prime}\left(\gamma_{1}\right)$ and to the root $v_{2}$ of $M^{\prime}\left(\gamma_{2}\right)$ (so that $v_{1,1}$ and $v_{2}$ become the left and the right child of $u_{1}$ in $M^{\prime}(\beta)$, respectively), and connect $u_{2}$ to the right root $v_{1,2}$ of $M^{\prime}\left(\gamma_{1}\right)$ and to the root $v_{3}$ of $M^{\prime}\left(\gamma_{3}\right)$ (so that $v_{1,2}$ and $v_{3}$ become the left and the right child of $u_{2}$ in $M^{\prime}(\beta)$, respectively). Note that the total cost of the new edges $\left(u_{1}, v_{1,1}\right),\left(u_{2}, v_{1,2}\right),\left(u_{1}, v_{2}\right)$, and $\left(u_{2}, v_{3}\right)$ in $M^{\prime}(\beta)$ is exactly the total weight of the edges between $\beta$ and its children in $\mathcal{D}(M)$. 
Type 2.3.3 (cf. Case 2.3.3 in Section 3.2): We create two root vertices $u_{1}$ and $u_{2}$ for $M^{\prime}(\beta)$, assign $u_{1}$ the first string in the string list $\mathcal{S}$ of $\beta$, assign $u_{2}$ the second string in $\mathcal{S}$, connect $u_{1}$ to the root $v_{1}$ of $M^{\prime}\left(\gamma_{1}\right)$ and to the right root $v_{2,2}$ of $M^{\prime}\left(\gamma_{2}\right)$ (so that $v_{1}$ and $v_{2,2}$ become the left and the right child of $u_{1}$ in $M^{\prime}(\beta)$, respectively), and connect $u_{2}$ to the left root $v_{2,1}$ of $M^{\prime}\left(\gamma_{2}\right)$ and to the root $v_{3}$ of $M^{\prime}\left(\gamma_{3}\right)$ (so that $v_{2,1}$ and $v_{3}$ become the left and the right child of $u_{2}$ in $M^{\prime}(\beta)$, respectively). Note that the total cost of the new edges $\left(u_{1}, v_{1}\right),\left(u_{2}, v_{2,1}\right),\left(u_{1}, v_{2,2}\right)$, and $\left(u_{2}, v_{3}\right)$ in $M^{\prime}(\beta)$ is exactly the total weight of the edges between $\beta$ and its children in $\mathcal{D}(M)$.

Type 2.3.4 (cf. Case 2.3.4 in Section 3.2): We create two root vertices $u_{1}$ and $u_{2}$ for $M^{\prime}(\beta)$, assign $u_{1}$ the first string in the strings list $\mathcal{S}$ of $\beta$, assign $u_{2}$ the second string in $\mathcal{S}$, connect $u_{1}$ to the root $v_{1}$ of $M^{\prime}\left(\gamma_{1}\right)$ and to the left root $v_{3,1}$ of $M^{\prime}\left(\gamma_{3}\right)$ (so that $v_{1}$ and $v_{3,1}$ become the left and the right child of $u_{1}$ in $M^{\prime}(\beta)$, respectively), and connect $u_{2}$ to the root $v_{2}$ of $M^{\prime}\left(\gamma_{2}\right)$ and to the right root $v_{3,2}$ of $M^{\prime}\left(\gamma_{3}\right)$ (so that $v_{2}$ and $v_{3,2}$ become the left and the right child of $u_{2}$ in $M^{\prime}(\beta)$, respectively). Note that the total cost of the new edges $\left(u_{1}, v_{1}\right),\left(u_{2}, v_{2}\right),\left(u_{1}, v_{3,1}\right)$, and $\left(u_{2}, v_{3,2}\right)$ in $M^{\prime}(\beta)$ is exactly the total weight of the edges between $\beta$ and its children in $\mathcal{D}(M)$.

Type 2.3.5 (cf. Case 2.3.5 in Section 3.2): We create two root vertices $u_{1}$ and $u_{2}$ for $M^{\prime}(\beta)$, assign $u_{1}$ the first string in the string list $\mathcal{S}$ of $\beta$, assign $u_{2}$ the second string in $\mathcal{S}$, connect $u_{1}$ to the left root $v_{1,1}$ and the right root $v_{1,3}$ of $M^{\prime}\left(\gamma_{1}\right)$ (so that $v_{1,1}$ and $v_{1,3}$ become the left and the right child of $u_{1}$ in $M^{\prime}(\beta)$, respectively), and connect $u_{2}$ to the middle root $v_{1,2}$ of $M^{\prime}\left(\gamma_{1}\right)$ and to the root $v_{2}$ of $M^{\prime}\left(\gamma_{2}\right)$ (so that $v_{1,2}$ and $v_{2}$ become the left and the right child of $u_{2}$ in $M^{\prime}(\beta)$, respectively). Note that the total cost of the new edges $\left(u_{1}, v_{1,1}\right),\left(u_{2}, v_{1,2}\right),\left(u_{1}, v_{1,3}\right)$, and $\left(u_{2}, v_{2}\right)$ in $M^{\prime}(\beta)$ is exactly the total weight of the edges between $\beta$ and its children in $\mathcal{D}(M)$.

Type 2.3.6 (cf. Case 2.3.6 in Section 3.2): We create two root vertices $u_{1}$ and $u_{2}$ for $M^{\prime}(\beta)$, assign $u_{1}$ the first string in string list $\mathcal{S}$ of $\beta$, assign $u_{2}$ the second string in $\mathcal{S}$, connect $u_{1}$ to the left root $v_{1,1}$ of $M^{\prime}\left(\gamma_{1}\right)$ and to the left root $v_{2,1}$ of $M^{\prime}\left(\gamma_{2}\right)$ (so that $v_{1,1}$ and $v_{2,1}$ become the left and the right child of $u_{1}$ in $M^{\prime}(\beta)$, respectively), and connect $u_{2}$ to the right root $v_{1,2}$ of $M^{\prime}\left(\gamma_{1}\right)$ and to the right root $v_{2,2}$ of $M^{\prime}\left(\gamma_{2}\right)$ (so that $v_{1,2}$ and $v_{2,2}$ become the left and the right child of $u_{2}$ in $M^{\prime}(\beta)$, respectively). Note that the total cost of the new edges $\left(u_{1}, v_{1,1}\right),\left(u_{2}, v_{1,2}\right),\left(u_{1}, v_{2,1}\right)$, and $\left(u_{2}, v_{2,2}\right)$ in $M^{\prime}(\beta)$ is exactly the total weight of the edges between $\beta$ and its children in $\mathcal{D}(M)$.

Type 2.3.7 (cf. Case 2.3.7 in Section 3.2): We create two root vertices $u_{1}$ and $u_{2}$ for $M^{\prime}(\beta)$, assign $u_{1}$ the first string in the string list $\mathcal{S}$ of $\beta$, assign $u_{2}$ the second string in $\mathcal{S}$, connect $u_{1}$ to the root $v_{1}$ of $M^{\prime}\left(\gamma_{1}\right)$ and to the middle root $v_{2,2}$ of $M^{\prime}\left(\gamma_{2}\right)$ (so that $v_{1}$ and $v_{2,2}$ become the left and the right child of $u_{1}$ in $M^{\prime}(\beta)$, respectively), and connect $u_{2}$ to the left root $v_{2,1}$ and the right root $v_{2,3}$ of $M^{\prime}\left(\gamma_{2}\right)$ (so that $v_{2,1}$ and $v_{2,3}$ become the left and the right child of $u_{2}$ in $M^{\prime}(\beta)$, respectively). Note that the total cost of the new edges $\left(u_{1}, v_{1}\right),\left(u_{2}, v_{2,1}\right),\left(u_{1}, v_{2,2}\right)$, and $\left(u_{2}, v_{2,3}\right)$ in $M^{\prime}(\beta)$ is exactly the total weight of the edges between $\beta$ and its children in $\mathcal{D}(M)$.

Type 2.3.8 (cf. Case 2.3.8 in Section 3.2): We create two root vertices $u_{1}$ and $u_{2}$ for $M^{\prime}(\beta)$, assign $u_{1}$ the first string in the string list $\mathcal{S}$ of $\beta$, assign $u_{2}$ the second string in $\mathcal{S}$, connect $u_{1}$ to the leftmost root $v_{1,1}$ and the third leftmost root $v_{1,3}$ of $M^{\prime}\left(\gamma_{1}\right)$ (so that $v_{1,1}$ and $v_{1,3}$ become the left and the right child of $u_{1}$ in $M^{\prime}(\beta)$, respectively), and connect $u_{2}$ to the second leftmost root $v_{1,2}$ and the rightmost root $v_{1,4}$ of $M^{\prime}\left(\gamma_{1}\right)$ (so that $v_{1,2}$ and $v_{1,4}$ become the left and the right child of $u_{2}$ in $M^{\prime}(\beta)$, respectively). Note that the total cost of the new edges $\left(u_{1}, v_{1,1}\right),\left(u_{2}, v_{1,2}\right),\left(u_{1}, v_{1,3}\right)$, and $\left(u_{2}, v_{1,4}\right)$ in $M^{\prime}(\beta)$ is exactly the total weight of the edges between $\beta$ and its children in $\mathcal{D}(M)$.

Type 3.1 (cf. Case 3.1 in Section 3.2): We connect $M^{\prime}\left(\gamma_{1}\right)$ and $M^{\prime}\left(\gamma_{2}\right)$ by making the right root 
$v_{1,2}$ of $M^{\prime}\left(\gamma_{1}\right)$ be the unique child of the leftmost leaf $v_{2,1}$ of $M^{\prime}\left(\gamma_{2}\right)$. Note that the cost of the new edge $\left(v_{2,1}, v_{1,2}\right)$ in $M^{\prime}(\beta)$ is exactly the total weight of the edges between $\beta$ and its children in $\mathcal{D}(M)$.

Type 3.2 (cf. Case 3.2 in Section 3.2): We connect $M^{\prime}\left(\gamma_{1}\right)$ and $M^{\prime}\left(\gamma_{2}\right)$ by making the leftmost root $v_{2,1}$ of $M^{\prime}\left(\gamma_{2}\right)$ be the unique child of the rightmost leaf $v_{1,2}$ of $M^{\prime}\left(\gamma_{1}\right)$. Note that the cost of the new edge $\left(v_{1,2}, v_{2,1}\right)$ in $M^{\prime}(\beta)$ is exactly the total weight of the edges between $\beta$ and its children in $\mathcal{D}(M)$.

Lemma 3.8 The cost of the new mode $M^{\prime}$ constructed from $\mathcal{D}(M)$ as above equals the total weight of edges in $\mathcal{D}(M)$. Consequently, $c\left(M^{\prime}\right) \leq 3 \cdot c(M)$.

Proof. We claim that for each node $\beta$ with a label $(\mathcal{S}, i, j)$ in $\mathcal{D}(M)$, the rooted ordered forest $M^{\prime}(\beta)$ constructed as above satisfies that $c\left(M^{\prime}(\beta)\right)$ equals the total weight of edges in $\mathcal{D}_{M}(\mathcal{S}, i, j)$ and the leaves of $M^{\prime}(\beta)$ are $s_{i}, s_{i+1}, \ldots, s_{j}$ (from left to right). The claim can be easily shown by induction on $j-i$ and so its proof is omitted. The claim implies the first assertion in the lemma immediately. The second assertion follows from the first and Lemma 3.7.

\subsection{Computing an Optimal Component Tree}

An obvious but very crucial property in the construction of $M^{\prime}$ from $\mathcal{D}(M)$ given in Section 3.3 is that the construction of $M^{\prime}$ only depends on the label and the type of each node in $\mathcal{D}(M)$. This motivates us to define component trees (independently of duplication models) for $\left\langle s_{1}, \ldots, s_{n}\right\rangle$ and to use dynamic programming to compute a minimum-weight component tree.

In order to define component trees (independently of duplication models) for $\left\langle s_{1}, \ldots, s_{n}\right\rangle$, we need to define several other terms first. An abstract quadruple is a quadruple $(\mathcal{S}, i, j, t)$, where $\mathcal{S}$ is an ordered nonempty list of at most four strings among $s_{1}, \ldots, s_{n}, i$ and $j$ are two integers with $1 \leq i \leq j \leq n$, and $t$ satisfies the following conditions:

- $t \in\{0,1.1,1.2,2.1,2.2,2.3 .1, \ldots, 2.3 .8,3.1,3.2\}$.

- If $t=0$, then $i=j$ and $\mathcal{S}=\left\{s_{i}\right\}$.

- If $t \in\{1.1,1.2\}$, then $|\mathcal{S}|=1$ and $j-i \geq 1$.

- If $t \in\{2.1,2.2,2.3 .1, \ldots, 2.3 .8\}$, then $|\mathcal{S}|=2$ and $j-i \geq 3$.

- If $t \in\{3.1,3.2\}$, then $3 \leq|\mathcal{S}| \leq 4$ and $j-i \geq 2|\mathcal{S}|-1$.

We call $t$ the type of the abstract quadruple $(\mathcal{S}, i, j, t)$. For convenience, if $t \in\{0,1.1,1.2\}$, we say that $(\mathcal{S}, i, j, t)$ is of type 1 ; if $t \in\{2.1,2.2,2.3 .1, \ldots, 2.3 .8\}$, we say that $(\mathcal{S}, i, j, t)$ is of type 2 ; if $t \in\{3.1,3.2\}$, we say that $(\mathcal{S}, i, j, t)$ is of type 3 .

We define component trees for abstract quadruples $(\mathcal{S}, i, j, t)$ by induction on $j-i$. In the base case where $j-i=0$, a component tree for $(\mathcal{S}, i, j, t)$ is a rooted tree with only one node (the root) which is labeled with $(\mathcal{S}, i, j)$ and is of type $t$.

Consider the case where $j-i \geq 1$. Suppose that $\mathcal{S}=\left\langle s_{h_{1}}, \ldots, s_{h_{g}}\right\rangle$. A component tree for $(\mathcal{S}, i, j, t)$ is a rooted ordered tree $\mathcal{D}$ such that the $\operatorname{root} \beta$ is labeled with $(\mathcal{S}, i, j)$ and is of type $t$, and the following conditions are satisfied: 
- If $t=1.1$, then $\beta$ has only one child in $\mathcal{D}$ and the subtree rooted at the child in $\mathcal{D}$ is a component tree for some type-2 abstract quadruple $\left(\mathcal{S}^{\prime}, i, j, t^{\prime}\right)$.

- If $t=1.2$, then $\beta$ has two children $\gamma_{1}$ and $\gamma_{2}$ in $\mathcal{D}$, the subtree rooted at $\gamma_{1}$ in $\mathcal{D}$ is a component tree for some type-1 abstract quadruple $\left(\mathcal{S}_{1}, i, k, t_{1}\right)$ with $k<j$, and the subtree rooted at $\gamma_{2}$ in $\mathcal{D}$ is a component tree for some type- 1 abstract quadruple $\left(\mathcal{S}_{2}, k+1, j, t_{2}\right)$.

- If $t=2.1$, then $\beta$ has two children $\gamma_{1}$ and $\gamma_{2}$ in $\mathcal{D}$, the subtree rooted at $\gamma_{1}$ in $\mathcal{D}$ is a component tree for some type-2 abstract quadruple $\left(\left\langle s_{h_{1}}\right\rangle, i, k, t_{1}\right)$ with $k<j$, and the subtree rooted at $\gamma_{2}$ in $\mathcal{D}$ is a component tree for some type-2 abstract quadruple $\left(\left\langle s_{x}, s_{h_{2}}\right\rangle, k, j, t_{2}\right)$ with $1 \leq x \leq n$.

- If $t=2.2$, then $\beta$ has two children $\gamma_{1}$ and $\gamma_{2}$ in $\mathcal{D}$, the subtree rooted at $\gamma_{1}$ in $\mathcal{D}$ is a component tree for some type-2 abstract quadruple $\left(\left\langle s_{h_{1}}, s_{x}\right\rangle, i, \ell, t_{1}\right)$ with $1 \leq x \leq n$ and $\ell<j$, and the subtree rooted at $\gamma_{2}$ in $\mathcal{D}$ is a component tree for some type- 2 abstract quadruple $\left(\left\langle s_{h_{2}}\right\rangle, \ell, j, t_{2}\right)$.

- If $t=2.3 .1$, then $\beta$ has four children $\gamma_{1}, \ldots, \gamma_{4}$ in $\mathcal{D}$, the subtree rooted at $\gamma_{1}$ in $\mathcal{D}$ is a component tree for some type-1 abstract quadruple $\left(\mathcal{S}_{1}, i, k_{1}, t_{1}\right)$ with $k_{1}<j$, the subtree rooted at $\gamma_{2}$ in $\mathcal{D}$ is a component tree for some type- 1 abstract quadruple $\left(\mathcal{S}_{2}, k_{1}+1, k_{2}, t_{2}\right)$ with $k_{2}<j$, the subtree rooted at $\gamma_{3}$ in $\mathcal{D}$ is a component tree for some type- 1 abstract quadruple $\left(\mathcal{S}_{3}, k_{2}+1, k_{3}, t_{3}\right)$ with $k_{3}<j$, and the subtree rooted at $\gamma_{4}$ in $\mathcal{D}$ is a component tree for some type-1 abstract quadruple $\left(\mathcal{S}_{4}, k_{3}+1, j, t_{4}\right)$.

- If $t=2.3 .2$, then $\beta$ has three children $\gamma_{1}, \ldots, \gamma_{3}$ in $\mathcal{D}$, the subtree rooted at $\gamma_{1}$ in $\mathcal{D}$ is a component tree for some type-2 abstract quadruple $\left(\mathcal{S}_{1}, i, k, t_{1}\right)$ with $k<j$, the subtree rooted at $\gamma_{2}$ in $\mathcal{D}$ is a component tree for some type- 1 abstract quadruple $\left(\mathcal{S}_{2}, k+1, \ell, t_{2}\right)$ with $\ell<j$, and the subtree rooted at $\gamma_{3}$ in $\mathcal{D}$ is a component tree for some type- 1 abstract quadruple $\left(\mathcal{S}_{3}, \ell+1, j, t_{3}\right)$.

- If $t=2.3 .3$, then $\beta$ has three children $\gamma_{1}, \ldots, \gamma_{3}$ in $\mathcal{D}$, the subtree rooted at $\gamma_{1}$ in $\mathcal{D}$ is a component tree for some type-1 abstract quadruple $\left(\mathcal{S}_{1}, i, k, t_{1}\right)$ with $k<j$, the subtree rooted at $\gamma_{2}$ in $\mathcal{D}$ is a component tree for some type-2 abstract quadruple $\left(\mathcal{S}_{2}, k+1, \ell, t_{2}\right)$ with $\ell<j$, and the subtree rooted at $\gamma_{3}$ in $\mathcal{D}$ is a component tree for some type-1 abstract quadruple $\left(\mathcal{S}_{3}, \ell+1, j, t_{3}\right)$.

- If $t=2.3 .4$, then $\beta$ has three children $\gamma_{1}, \ldots, \gamma_{3}$ in $\mathcal{D}$, the subtree rooted at $\gamma_{1}$ in $\mathcal{D}$ is a component tree for some type-1 abstract quadruple $\left(\mathcal{S}_{1}, i, k, t_{1}\right)$ with $k<j$, the subtree rooted at $\gamma_{2}$ in $\mathcal{D}$ is a component tree for some type- 1 abstract quadruple $\left(\mathcal{S}_{2}, k+1, \ell, t_{2}\right)$ with $\ell<j$, and the subtree rooted at $\gamma_{3}$ in $\mathcal{D}$ is a component tree for some type-2 abstract quadruple $\left(\mathcal{S}_{3}, \ell+1, j, t_{3}\right)$.

- If $t=2.3 .5$, then $\beta$ has two children $\gamma_{1}$ and $\gamma_{2}$ in $\mathcal{D}$, the subtree rooted at $\gamma_{1}$ in $\mathcal{D}$ is a component tree for some type-3 abstract quadruple $\left(\mathcal{S}_{1}, i, k, t_{1}\right)$ with $\left|\mathcal{S}_{1}\right|=3$ and $k<j$, and the subtree rooted at $\gamma_{2}$ in $\mathcal{D}$ is a component tree for some type-1 abstract quadruple $\left(\mathcal{S}_{2}, k+1, j, t_{2}\right)$. 
- If $t=2.3 .6$, then $\beta$ has two children $\gamma_{1}$ and $\gamma_{2}$ in $\mathcal{D}$, the subtree rooted at $\gamma_{1}$ in $\mathcal{D}$ is a component tree for some type-2 abstract quadruple $\left(\mathcal{S}_{1}, i, k, t_{1}\right)$ with $k<j$, and the subtree rooted at $\gamma_{2}$ in $\mathcal{D}$ is a component tree for some type-2 abstract quadruple $\left(\mathcal{S}_{2}, k+1, j, t_{2}\right)$.

- If $t=2.3 .7$, then $\beta$ has two children $\gamma_{1}$ and $\gamma_{2}$ in $\mathcal{D}$, the subtree rooted at $\gamma_{1}$ in $\mathcal{D}$ is a component tree for some type-1 abstract quadruple $\left(\mathcal{S}_{1}, i, k, t_{1}\right)$ with $k<j$, and the subtree rooted at $\gamma_{2}$ in $\mathcal{D}$ is a component tree for some type-3 abstract quadruple $\left(\mathcal{S}_{2}, k+1, j, t_{2}\right)$ with $\left|\mathcal{S}_{2}\right|=3$.

- If $t=2.3 .8$, then $\beta$ has only one child in $\mathcal{D}$ and the subtree rooted at the child in $\mathcal{D}$ is a component tree for some type-3 abstract quadruple $\left(\mathcal{S}^{\prime}, i, j, t^{\prime}\right)$ with $\left|\mathcal{S}^{\prime}\right|=4$.

- If $t=3.1$, then $\beta$ has two children $\gamma_{1}$ and $\gamma_{2}$ in $\mathcal{D}$, the subtree rooted at $\gamma_{1}$ in $\mathcal{D}$ is a component tree for some type-2 abstract quadruple $\left(\left\langle s_{h_{1}}, s_{x}\right\rangle, i, k, t_{1}\right)$ with $1 \leq x \leq n$ and $k<j$, and the subtree rooted at $\gamma_{2}$ in $\mathcal{D}$ is a component tree for some type- $(g-1)$ abstract quadruple $\left(\left\langle s_{h_{2}}, \ldots, s_{h_{g}}\right\rangle, k, j, t_{2}\right)$.

- If $t=3.2$, then $\beta$ has two children $\gamma_{1}$ and $\gamma_{2}$ in $\mathcal{D}$, the subtree rooted at $\gamma_{1}$ in $\mathcal{D}$ is a component tree for some type-2 abstract quadruple $\left(\left\langle s_{h_{1}}, s_{h_{2}}\right\rangle, i, k, t_{1}\right)$ with $k<j$, and the subtree rooted at $\gamma_{2}$ in $\mathcal{D}$ is a component tree for some type- $(g-1)$ abstract quadruple $\left(\left\langle s_{x}, s_{h_{3}}, \ldots, s_{h_{g}}\right\rangle, k, j, t_{2}\right)$ with $1 \leq x \leq n$.

Let $(\mathcal{S}, i, j, t)$ be an abstract quadruple, and let $\mathcal{D}$ be a component tree for $(\mathcal{S}, i, j, t)$. Note that each node in $\mathcal{D}$ is labeled with a triple $(L, k, \ell)$, where $L$ is an ordered nonempty list of strings among $s_{1}, \ldots, s_{n}$ and $k$ and $\ell$ are integers with $1 \leq k \leq \ell \leq n$. Moreover, each node in $\mathcal{D}$ has a type among $0,1.1,1.2,2.1,2.2,2.3 .1, \ldots, 2.3 .8,3.1,3.2$. Thus, we can use $\mathcal{D}$ to construct a rooted ordered forest $M_{\mathcal{D}}^{\prime}$ with $|\mathcal{S}|$ roots as described in Section 3.3. We define the weight of $\mathcal{D}$ to be $\sum_{e} c(e)$, where $e$ ranges over all edges in $M_{\mathcal{D}}^{\prime}$ and $c(e)$ is the hamming distance between the two strings assigned to the endpoints of $e$ in $M_{\mathcal{D}}^{\prime}$.

We are now ready to use dynamic programming to compute a minimum-weight component tree for each abstract quadruple $(\mathcal{S}, i, j, t)$. For simplicity, we only explicitly give formulas for computing the minimum weight $W(\mathcal{S}, i, j, t)$ of a component tree for each abstract quadruple $(\mathcal{S}, i, j, t)$ as follows.

- For each abstract quadruple $q=(\mathcal{S}, i, j, t)$ with $t=0, W(q)=0$.

- For each abstract quadruple $q=\left(\left\langle s_{h}\right\rangle, i, j, t\right)$ with $t=1.1$,

$$
W(q)=\min _{q^{\prime}} W\left(q^{\prime}\right)+d\left(s_{h}, s_{x}\right)+d\left(s_{h}, s_{y}\right),
$$

where $q^{\prime}$ ranges over all type-2 abstract quadruples $\left(\left\langle s_{x}, s_{y}\right\rangle, i, j, t^{\prime}\right)$.

- For each abstract quadruple $q=\left(\left\langle s_{h}\right\rangle, i, j, t\right)$ with $t=1.2$,

$$
W(q)=\min _{i \leq k<j} \min _{q_{1}} \min _{q_{2}} W\left(q_{1}\right)+W\left(q_{2}\right)+d\left(s_{h}, s_{x}\right)+d\left(s_{h}, s_{y}\right),
$$

where $q_{1}$ ranges over all type- 1 abstract quadruples $\left(\left\langle s_{x}\right\rangle, i, k, t_{1}\right)$ and $q_{2}$ ranges over all type-1 abstract quadruples $\left(\left\langle s_{y}\right\rangle, k+1, j, t_{2}\right)$. 
- For each abstract quadruple $q=\left(\left\langle s_{h_{1}}, s_{h_{2}}\right\rangle, i, j, t\right)$ with $t=2.1$,

$$
W(q)=\min _{i<k<j} \min _{q_{1}} \min _{q_{2}} W\left(q_{1}\right)+W\left(q_{2}\right)+d\left(s_{k}, s_{x}\right),
$$

where $q_{1}$ ranges over all type- 1 abstract quadruples $\left(\left\langle s_{h_{1}}\right\rangle, i, k, t_{1}\right)$ and $q_{2}$ ranges over all type-2 abstract quadruples $\left(\left\langle s_{x}, s_{h_{2}}\right\rangle, k, j, t_{2}\right)$.

- For each abstract quadruple $q=\left(\left\langle s_{h_{1}}, s_{h_{2}}\right\rangle, i, j, t\right)$ with $t=2.2$,

$$
W(q)=\min _{i<\ell<j} \min _{q_{1}} \min _{q_{2}} W\left(q_{1}\right)+W\left(q_{2}\right)+d\left(s_{\ell}, s_{x}\right),
$$

where $q_{1}$ ranges over all type- 2 abstract quadruples $\left(\left\langle s_{h_{1}}, s_{x}\right\rangle, i, \ell, t_{1}\right)$ and $q_{2}$ ranges over all type-1 abstract quadruples $\left(\left\langle s_{h_{2}}\right\rangle, \ell, j, t_{2}\right)$.

- For each abstract quadruple $q=\left(\left\langle s_{h_{1}}, s_{h_{2}}\right\rangle, i, j, t\right)$ with $t=2.3 .1$,

$$
\begin{aligned}
W(q) & =\min _{i \leq k_{1}<j} \min _{k_{1}<k_{2}<j} \min _{k_{2}<k_{3}<j} \min _{q_{1}} \min _{q_{2}} \min _{q_{3}} \min _{q_{4}} W\left(q_{1}\right)+W\left(q_{2}\right)+W\left(q_{3}\right)+W\left(q_{4}\right) \\
& +d\left(s_{h_{1}}, s_{x_{1}}\right)+d\left(s_{h_{1}}, s_{x_{3}}\right)+d\left(s_{h_{2}}, s_{x_{2}}\right)+d\left(s_{h_{2}}, s_{x_{4}}\right),
\end{aligned}
$$

where $q_{1}$ ranges over all type- 1 abstract quadruples $\left(\left\langle s_{x_{1}}\right\rangle, i, k_{1}, t_{1}\right), q_{2}$ ranges over all type1 abstract quadruples $\left(\left\langle s_{x_{2}}\right\rangle, k_{1}+1, k_{2}, t_{2}\right), q_{3}$ ranges over all type-1 abstract quadruples $\left(\left\langle s_{x_{3}}\right\rangle, k_{2}+1, k_{3}, t_{3}\right)$, and $q_{4}$ ranges over all type- 1 abstract quadruples $\left(\left\langle s_{x_{4}}\right\rangle, k_{3}+1, k_{4}, t_{4}\right)$.

- For each abstract quadruple $q=\left(\left\langle s_{h_{1}}, s_{h_{2}}\right\rangle, i, j, t\right)$ with $t=2.3 .2$,

$$
\begin{aligned}
W(q) & =\min _{i+3 \leq k_{1}<j} \min _{k_{1}<k_{2}<j} \min _{q_{1}} \min _{q_{2}} \min _{q_{3}} W\left(q_{1}\right)+W\left(q_{2}\right)+W\left(q_{3}\right) \\
& +d\left(s_{h_{1}}, s_{x_{1}}\right)+d\left(s_{h_{1}}, s_{x_{3}}\right)+d\left(s_{h_{2}}, s_{x_{2}}\right)+d\left(s_{h_{2}}, s_{x_{4}}\right),
\end{aligned}
$$

where $q_{1}$ ranges over all type- 2 abstract quadruples $\left(\left\langle s_{x_{1}}, s_{x_{2}}\right\rangle, i, k_{1}, t_{1}\right), q_{2}$ ranges over all type1 abstract quadruples $\left(\left\langle s_{x_{3}}\right\rangle, k_{1}+1, k_{2}, t_{2}\right)$, and $q_{3}$ ranges over all type- 1 abstract quadruples $\left(\left\langle s_{x_{4}}\right\rangle, k_{2}+1, j, t_{3}\right)$.

- For each abstract quadruple $q=\left(\left\langle s_{h_{1}}, s_{h_{2}}\right\rangle, i, j, t\right)$ with $t=2.3 .3$,

$$
\begin{aligned}
W(q) & =\min _{i \leq k_{1}<j} \min _{k_{1}+4 \leq k_{2}<j} \min _{q_{1}} \min _{q_{2}} \min _{q_{3}} W\left(q_{1}\right)+W\left(q_{2}\right)+W\left(q_{3}\right) \\
& +d\left(s_{h_{1}}, s_{x_{1}}\right)+d\left(s_{h_{1}}, s_{x_{3}}\right)+d\left(s_{h_{2}}, s_{x_{2}}\right)+d\left(s_{h_{2}}, s_{x_{4}}\right),
\end{aligned}
$$

where $q_{1}$ ranges over all type- 1 abstract quadruples $\left(\left\langle s_{x_{1}}\right\rangle, i, k_{1}, t_{1}\right), q_{2}$ ranges over all type-2 abstract quadruples $\left(\left\langle s_{x_{2}}, s_{x_{3}}\right\rangle, k_{1}+1, k_{2}, t_{2}\right)$, and $q_{3}$ ranges over all type-1 abstract quadruples $\left(\left\langle s_{x_{4}}\right\rangle, k_{2}+1, j, t_{3}\right)$.

- For each abstract quadruple $q=\left(\left\langle s_{h_{1}}, s_{h_{2}}\right\rangle, i, j, t\right)$ with $t=2.3 .4$,

$$
\begin{aligned}
W(q) & =\min _{i \leq k_{1}<j} \min _{k_{1}<k_{2}<j} \min _{q_{1}} \min _{q_{2}} \min _{q_{3}} W\left(q_{1}\right)+W\left(q_{2}\right)+W\left(q_{3}\right) \\
& +d\left(s_{h_{1}}, s_{x_{1}}\right)+d\left(s_{h_{1}}, s_{x_{3}}\right)+d\left(s_{h_{2}}, s_{x_{2}}\right)+d\left(s_{h_{2}}, s_{x_{4}}\right),
\end{aligned}
$$

where $q_{1}$ ranges over all type- 1 abstract quadruples $\left(\left\langle s_{x_{1}}\right\rangle, i, k_{1}, t_{1}\right), q_{2}$ ranges over all type-1 abstract quadruples $\left(\left\langle s_{x_{2}}\right\rangle, k_{1}+1, k_{2}, t_{2}\right)$, and $q_{3}$ ranges over all type-2 abstract quadruples $\left(\left\langle s_{x_{3}}, s_{x_{4}}\right\rangle, k_{2}+1, j, t_{3}\right)$. 
- For each abstract quadruple $q=\left(\left\langle s_{h_{1}}, s_{h_{2}}\right\rangle, i, j, t\right)$ with $t=2.3 .5$,

$$
\begin{aligned}
W(q) & =\min _{i+5 \leq k<j} \min _{q_{1}} \min _{q_{2}} W\left(q_{1}\right)+W\left(q_{2}\right) \\
& +d\left(s_{h_{1}}, s_{x_{1}}\right)+d\left(s_{h_{1}}, s_{x_{3}}\right)+d\left(s_{h_{2}}, s_{x_{2}}\right)+d\left(s_{h_{2}}, s_{x_{4}}\right),
\end{aligned}
$$

where $q_{1}$ ranges over all type-3 abstract quadruples $\left(\left\langle s_{x_{1}}, s_{x_{2}}, s_{x_{3}}\right\rangle, i, k, t_{1}\right)$ and $q_{2}$ ranges over all type-1 abstract quadruples $\left(\left\langle s_{x_{4}}\right\rangle, k+1, j, t_{2}\right)$.

- For each abstract quadruple $q=\left(\left\langle s_{h_{1}}, s_{h_{2}}\right\rangle, i, j, t\right)$ with $t=2.3 .6$,

$$
\begin{aligned}
W(q) & =\min _{i+3 \leq k<j} \min _{q_{1}} \min _{q_{2}} W\left(q_{1}\right)+W\left(q_{2}\right) \\
& +d\left(s_{h_{1}}, s_{x_{1}}\right)+d\left(s_{h_{1}}, s_{x_{3}}\right)+d\left(s_{h_{2}}, s_{x_{2}}\right)+d\left(s_{h_{2}}, s_{x_{4}}\right),
\end{aligned}
$$

where $q_{1}$ ranges over all type-2 abstract quadruples $\left(\left\langle s_{x_{1}}, s_{x_{2}}\right\rangle, i, k, t_{1}\right)$ and $q_{2}$ ranges over all type-2 abstract quadruples $\left(\left\langle s_{x_{3}}, s_{x_{4}}\right\rangle, k+1, j, t_{2}\right)$.

- For each abstract quadruple $q=\left(\left\langle s_{h_{1}}, s_{h_{2}}\right\rangle, i, j, t\right)$ with $t=2.3 .7$,

$$
\begin{aligned}
W(q) & =\min _{i \leq k<j} \min _{q_{1}} \min _{q_{2}} W\left(q_{1}\right)+W\left(q_{2}\right) \\
& +d\left(s_{h_{1}}, s_{x_{1}}\right)+d\left(s_{h_{1}}, s_{x_{3}}\right)+d\left(s_{h_{2}}, s_{x_{2}}\right)+d\left(s_{h_{2}}, s_{x_{4}}\right),
\end{aligned}
$$

where $q_{1}$ ranges over all type- 1 abstract quadruples $\left(\left\langle s_{x_{1}}\right\rangle, i, k, t_{1}\right)$ and $q_{2}$ ranges over all type-3 abstract quadruples $\left(\left\langle s_{x_{2}}, s_{x_{3}}, s_{x_{4}}\right\rangle, k+1, j, t_{2}\right)$.

- For each abstract quadruple $q=\left(\left\langle s_{h_{1}}, s_{h_{2}}\right\rangle, i, j, t\right)$ with $t=2.3 .8$,

$$
W(q)=\min _{q^{\prime}} W\left(q^{\prime}\right)+d\left(s_{h_{1}}, s_{x_{1}}\right)+d\left(s_{h_{1}}, s_{x_{3}}\right)+d\left(s_{h_{2}}, s_{x_{2}}\right)+d\left(s_{h_{2}}, s_{x_{4}}\right),
$$

where $q^{\prime}$ ranges over all type-3 abstract quadruples $\left(\left\langle s_{x_{1}}, \ldots, s_{x_{4}}\right\rangle, i, j, t^{\prime}\right)$.

- For each abstract quadruple $q=\left(\left\langle s_{h_{1}}, \ldots, s_{h_{g}}\right\rangle, i, j, t\right)$ with $t=3.1$,

$$
W(q)=\min _{i+3 \leq k<j} \min _{q_{1}} \min _{q_{2}} W\left(q_{1}\right)+W\left(q_{2}\right)+d\left(s_{k}, s_{x}\right),
$$

where $q_{1}$ ranges over all type- 2 abstract quadruples $\left(\left\langle s_{h_{1}}, s_{x}\right\rangle, i, k, t_{1}\right)$ and $q_{2}$ ranges over all type- $(g-1)$ abstract quadruples $\left(\left\langle s_{h_{2}}, \ldots, s_{h_{g}}\right\rangle, k, j, t_{2}\right)$.

- For each abstract quadruple $q=\left(\left\langle s_{h_{1}}, \ldots, s_{h_{g}}\right\rangle, i, j, t\right)$ with $t=3.2$,

$$
W(q)=\min _{i+3 \leq k<j} \min _{q_{1}} \min _{q_{2}} W\left(q_{1}\right)+W\left(q_{2}\right)+d\left(s_{k}, s_{x}\right),
$$

where $q_{1}$ ranges over all type-2 abstract quadruples $\left(\left\langle s_{h_{1}}, s_{h_{2}}\right\rangle, i, k, t_{1}\right)$ and $q_{2}$ ranges over all type- $(g-1)$ abstract quadruples $\left(\left\langle s_{x}, s_{h_{3}}, \ldots, s_{h_{g}}\right\rangle, k, j, t_{2}\right)$.

Obviously, the minimum weight of a component tree for $\left\langle s_{1}, \ldots, s_{n}\right\rangle$ is $\min _{q} W(q)$, where $q$ ranges over all type- 1 abstract quadruples $(\mathcal{S}, 1, n, t)$. Moreover, a rough estimate gives a running time of $O\left(n^{11}+m n^{2}\right)$. Indeed, we can lower the time complexity to $O\left(n^{8}+m n^{2}\right)$ by modifying the above dynamic programming appropriately (although we omit the details here to save space). Thus, we have the following lemma: 
Lemma 3.9 We can compute a minimum-weight component tree for $\left\langle s_{1}, \ldots, s_{n}\right\rangle$ in polynomial time.

Combining Lemmas 3.1, 3.8, and 3.9, we have the following theorem:

Theorem 3.10 There is a polynomial-time approximation algorithm for 2-DHR that achieves a ratio of 6 .

\section{Acknowledgements}

Zhi-Zhong Chen is supported in part by the Grant-in-Aid for Scientific Research of the Ministry of Education, Science, Sports and Culture of Japan, under Grant No. 17500012. Lusheng Wang and Zhanyong Wang are fully supported by a grant from the Research Grants Council of the Hong Kong Special Administrative Region, China [Project No. CityU 120905].

\section{References}

[1] G. Benson and L. Dong. Reconstructing the Duplication History of a Tandem Repeat. Proceedings of the Seventh International Conference on Intelligent Systems for Molecular Biology (ISMB-99), pp. 44-53, 1999.

[2] T. Boby, A. -M. Patch, and S. J. Aves. TRbase: A Database Relating Tandem Repeats to Disease Genes for the Human Genome, Bioinformatics, Advance Access published on October 12, 2004.

[3] W. Fitch. Phylogenies Constrained by Cross-over Process as Illustrated by Human Hemoglobins in a Thirteen Cycle, Eleven Amino-Acid Repeat in Human Apolipoprotein A-I. Genetics, Vol. 86, pp. 623644, 1977.

[4] D. Jaitly, P. E. Kearney, G. Lin, and B. Ma. Methods for Reconstructing the History of Tandem Repeats and Their Application to the Human Genome. J. Comput. Syst. Sci., Vol. 65 , pp. 494-507, 2002.

[5] F. Lillo, S. Basile, and R. N. Mantegna. Comparative Genomics Study of Inverted Repeats in Bacteria. Bioinformatics, Vol. 18, pp. 971 - 979, 2002.

[6] J. Macas, T. Mszros, and M. Nouzov. PlantSat: A Specialized Database for Plant Satellite Repeats. Bioinformatics, Vol. 18, pp. 28 - 35, 2002.

[7] H. H. Otu and K. Sayood. A New Sequence Distance Measure for Phylogenetic Tree Construction. Bioinformatics, Vol. 19, pp. 2122 - 2130, 2004.

[8] S. Subramanian, R. K. Mishra, and L. Singh. Genome-Wide Analysis of Bkm Sequences (GATA Repeats): Predominant Association with Sex Chromosomes and Potential Role in Higher Order Chromatin Organization and Function. Bioinformatics, Vol. 19, pp. 681-685, 2003.

[9] M. Tang, M. S. Waterman, and S. Yooseph. Zinc Finger Gene Clusters and Tandem Gene Duplication. Journal of Computational Biology, Vol. 9, pp. 429-446, 2002.

[10] L. Wang, T. Jiang, and E. L. Lawler. Approximation Algorithms for Tree Alignment with a Given Phylogeny. Algorithmica, Vol. 16, pp. 302-315, 1996.

[11] B. Widegren, U. Arnason, and G. Akusjarvi. Characteristics of a Conserved 1,579-bp Highly Repetitive Component in the Killer Whale, Ornicus Orca. Mol. Biol. Evol., Vol. 2, pp. 411-419, 1985. 
[12] A. H. Wyman and R. White. A Highly Polymorphic Locus in Human DNA. Proc. Natl. Acad. Sci., Vol. 77, pp. 6745-6758, 1980.

[13] L. Zhang, B. Ma, L. Wang, and Y. Xu. Greedy Method for Inferring Tandem Duplication History. Bioinformatics, Vol. 19, pp. 1497-1504, 2003. 